\title{
Nanoscale
}

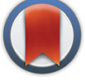

CrossMark

\& click for updates

Cite this: Nanoscale, 2014, 6, 13283

Received 1st July 2014

Accepted 8th September 2014

DOI: 10.1039/c4nr03670k

www.rsc.org/nanoscale

\section{Nanoelectronic circuits based on two-dimensional atomic layer crystals}

\author{
Seunghyun Lee ${ }^{a}$ and Zhaohui Zhong ${ }^{b}$
}

Since the discovery of graphene and related forms of two-dimensional (2D) atomic layer crystals, numerous studies have reported on the fundamental material aspects, such as the synthesis, the physical properties, and the electrical properties on the transistor level. With the advancement in large-area synthesis methods, system level integration to exploit the unique applications of these materials is close at hand. The main purpose of this review is to focus on the current progress and the prospect of circuits and systems based on 2D material that go beyond the single-transistor level studies. Both analog and digital circuits based on graphene and related 2D atomic layer crystals will be discussed.

\section{Introduction}

The study of 2D atomic layer crystals has garnered great interest in the electronics community. ${ }^{1-6}$ Materials such as graphene, boron nitride, monolayer transition metal dichalcogenides (TMDs), and various functionalized forms of graphene exhibit remarkable properties that can be exploited for many applications. ${ }^{1,3,4,6-8}$ Hence, there have been tremendous efforts to understand the fundamental properties of

${ }^{a}$ Center for Integrated Systems, Department of Electrical Engineering, Stanford University, Stanford, California 94305, USA. E-mail: seansl@stanford.edu; Tel: $+734-709-4936$

${ }^{b}$ Department of Electrical Engineering and Computer Science, University of Michigan, Ann Arbor, Michigan 48109, USA. E-mail: zzhong@umich.edu; Tel: +734-647-1953 these $2 \mathrm{D}$ crystals across various scientific disciplines. ${ }^{1,2,5,7,9-11}$ For these purposes, the bulk of research has been conducted with mechanically exfoliated samples with no possible route for large-scale integration. However, recent advancement in large-scale synthesis methods has opened up new doors for novel electronic applications. For graphene, the development of the large-area chemical vapour deposition (CVD) synthesis method $^{12-21}$ led to explosive growth in the research findings and diverse routes for commercial applications. Several synthesis methods for monolayer $\mathrm{MoS}_{2}$, a semiconducting TMD, based on chemical vapour deposition and vapour phase growth methods have also been reported. ${ }^{22-28}$

The main focus of this work is to understand the current progress and the prospect of large-scale integrations, and "circuits", which goes beyond the single transistor level

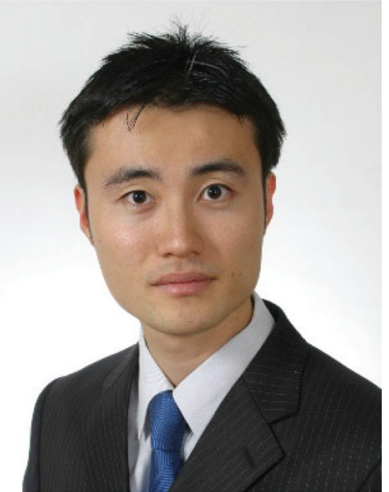

Seunghyun Lee
Seunghyun Lee received his B.S. degree from the Seoul National University in Korea, and the M.S. and Ph.D. degrees from the University of Michigan, Ann Arbor in 2013. He is currently a postdoctoral scholar at Stanford University. His research focuses on novel nano-electronic components/circuits based on low dimensional nanomaterials such as graphene and layered transition metal dichalcogenides. He is a recipient of the Gold Award from the Material Research Society (MRS 2012) and the 1st place award in the Texas Instrument analog circuit design contest (2008).

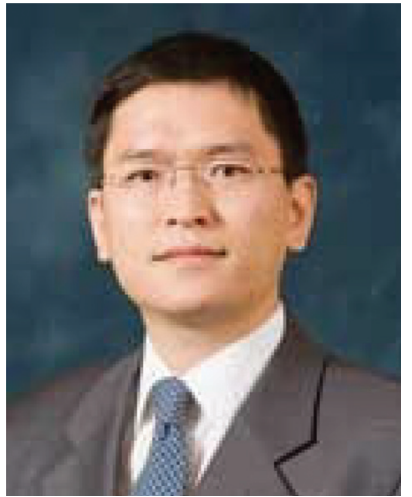

Zhaohui Zhong received his B.S. and M.S. from Nanjing University in 1998 and 2000, respectively, and his Ph.D. from Harvard University in 2005. From 2005 to 2008, he was a postdoctoral associate at Cornell Center for Nanoscale Systems. He joined the faculty of the University of Michigan in 2008. Currently he is an Associate Professor in the Department of Electrical Engineering and Computer Science at the University of Michigan. His research lies on the frontiers of nanomaterials, nanoelectronics, and nanophotonics. 
studies $^{2,8,29-33}$ of $2 \mathrm{D}$ materials. The primary goal is to understand the role of these emerging $2 \mathrm{D}$ materials in today's silicon dominated electronics industry.

The review is structured as follows: first, the electronic properties of $2 \mathrm{D}$ materials will be elucidated to understand the rationale behind the current interest in these materials. Then we will explain how graphene, the best known 2D atomic layer crystal, has found its way to improving various forms of analog and digital electronic systems: RF/mixed signal, ${ }^{34-46}$ hybrid-CMOS integration, ${ }^{39,40,44,47}$ logic, ${ }^{48-50}$ and passive components $^{47,51-54}$ (e.g. interconnects). Much of the progress was made possible because of the large-scale synthesis capability of the material. Next, the recent emergence of novel monolayer TMD materials with bandgaps, and the importance of these materials, will also be discussed, along with the recent progress on the integration of TMD-based circuits. ${ }^{55-58}$ Finally, we conclude with perspectives on the future of these $2 \mathrm{D}$ materials in electronics.

\section{The motivations to use 2D materials in electronics}

2D materials share several common traits due to their extremely thin profile. One of their most attractive attributes is the potential to facilitate transistor scaling. As transistor feature lengths are reaching sub-10 $\mathrm{nm}$ regimes, subsequent scaling will soon approach limits due to short-channel effects and statistical quantum effects. The shorter gate length and thinner gate oxide also require a thin semiconducting channel for transistor operation. ${ }^{59-61}$ With their atomically thin profile, these 2D materials have the potential to be scaled down for highspeed transistors without the adverse effects related to dimensional scaling. ${ }^{8}$

Moreover, with the recent progress in large-scale synthesis, some of these $2 \mathrm{D}$ materials can be readily transferred and stacked for large-scale integration. ${ }^{28,52}$ The low temperature transfer capability and the ease of stacking indicate the possibilities for higher integration density in the out-of-plane direction. This can be a major advantage in today's threedimensionally stacked circuit structure. ${ }^{62-64}$

Another notable trait is that the electrons of the constituent atoms of these materials (carbon atom for graphene and chalcogen atoms for TMDs) are terminated at the surface of the layers, and the absence of the dangling bonds allows these layers to be very stable in the environment. ${ }^{1}$ For extremely thin channels formed from bulk materials, random thickness variations and the dangling bonds were found to be major impediments that cause severe performance reduction, such as mobility degradation ${ }^{59-61}$ and threshold voltage shifts. ${ }^{59} 2 \mathrm{D}$ materials, on the other hand, can form an extremely smooth and thin channel material without the random thickness fluctuation. ${ }^{1,8}$

Despite the similarities resulting from a thin, layered atomic configuration, the electronic properties of $2 \mathrm{D}$ materials can be very different depending on the stoichiometry and the crystalline structure. For example, graphene is a zero bandgap semimetal $^{4,5,9}$ while some TMDs have sizable $(>1 \mathrm{eV})$ bandgaps. ${ }^{65,66}$ Materials such as boron nitride ${ }^{67}$ and fluorographene ${ }^{68}$ are effective wide-bandgap insulators. With such a diverse choice of available materials, it is imperative to understand their unique properties to investigate possible applications.

\section{Electronic properties of graphene}

Graphene atoms are arranged in a hexagonal crystal lattice as shown in Fig. 1a. The structure can be understood as a triangular lattice with a basis of two atoms per unit cell (depicted as blue and yellow in Fig. 1a). The band structure of graphene can be derived from a simple nearest-neighbour tight-binding approximation. ${ }^{70}$ Since graphene has two atoms per unit cell, the points of particular importance are $\mathrm{K}$ and $\mathrm{K}^{\prime}$ points at the corners of the graphene Brillouin zone (Fig. 1b). Near these points, the energy bands derived from the tight-binding Hamiltonian shows linear dependence of electron energy to the wave vector ${ }^{71,72}$ as shown in the zoom-in of Fig. 1c. The bandgap of graphene is exactly zero, with the conduction band and valence band meeting at the $\mathrm{K}$ and $\mathrm{K}^{\prime}$ points, also known as Dirac points or charge neutrality points.

The most interesting aspect of the graphene energy spectrum is that its charge carriers can be described by a Dirac spectrum for massless fermions ${ }^{73}$ rather than the usual Schrödinger equation for nonrelativistic particles. ${ }^{5,9}$ The quasiparticles in graphene exhibit a linear dispersion relation following the equation $E=\hbar k v_{\mathrm{F}}$, as if they were massless relativistic particles governed by the Dirac equation. ${ }^{5,9}$ (Here, the role of the speed of light $c$ is replaced by Fermi velocity $v_{\mathrm{F}} \approx c / 300$.) This means that electrons in graphene all move at (a)

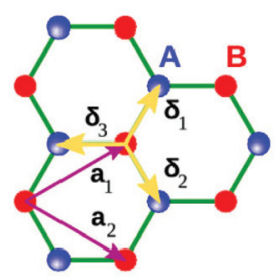

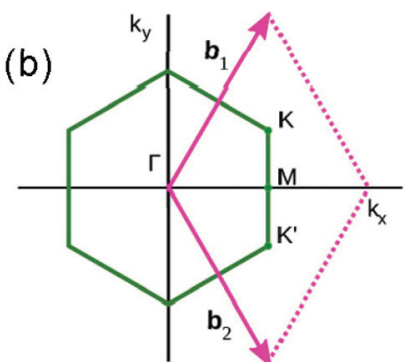

(c)

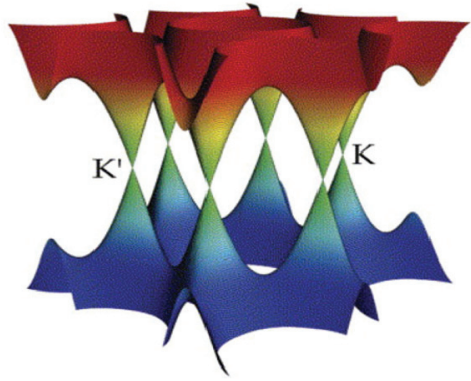

Fig. 1 (a) Hexagonal crystal lattice of graphene. $a_{1}$ and $a_{2}$ are the lattice unit vectors, and $\delta_{\mathrm{i}}, i=1,2,3$ are the nearest-neighbour vectors. (b) Graphene's Brillouin zone. The Dirac cones are located at the $K$ and $K^{\prime}$ points. (c) The energy dispersions of graphene crystal lattice. The conductance band touches the valence band at the $\mathrm{K}$ and the $\mathrm{K}^{\prime}$ points (i.e. the Dirac points). (Taken from ref. 69. Open access from Elsevier.) 
a constant velocity $\left(\sim v_{\mathrm{F}}\right)$ regardless of their momentum. Because of this linear dispersion, the quasiparticles in graphene behave very differently from other semiconductors or metals, with an energy spectrum approximated by parabolic (i.e. free electron-like) dispersion relations.

For example, although the bandgap is zero, the gate voltage can still modulate the density of states in graphene ${ }^{74}$ and switch from low conductivity states near the Dirac point to high conductivity states elsewhere (Fig. 2). However, because there is no bandgap, there is still a finite amount of current even at the low conductivity state near the Dirac point, ${ }^{74-76}$ leading to high switch-off current in graphene-based transistors. The minimum conductivity is also affected by defects, impurities and the chiral nature of electrons in graphene. ${ }^{10,75,76}$

The graphene crystal also shows exceptional electronic qualities such that charge carriers can travel ballistically over a submicron distance..$^{5,9,10,77}$ Room temperature mobility values exceeding $100000 \mathrm{~cm}^{2} \mathrm{~V}^{-1} \mathrm{~s}^{-1}$ have been reported in the literature. ${ }^{10,78-80}$ The mobility in these samples is limited by scattering on charged impurities ${ }^{81}$ or microscopic ripples. ${ }^{9,82}$ However, both sources of scattering can be reduced significantly by careful sample preparation, and they are not the ultimate limiting factors of carrier mobility in the graphene structure. ${ }^{77}$ It is the intrinsic scatterer such as the phonon, which cannot be removed at room temperature that sets the fundamental limit of mobility in graphene. ${ }^{10,77}$

Graphene's carrier transfer characteristic also stands out as it shows a perfect ambipolar electric field effect so that its charge carrier can be tuned continuously as shown in Fig. 2. 5,9,72 Its low-energy spectra are shown as insets in Fig. 2, indicating the changes in the position of the Fermi energy $E_{\mathrm{F}}$ with respect to the gate voltage $V_{\mathrm{g}}$. Positive gate voltage induces electrons while negative voltage induces holes. With its unique ambipolar transport properties and exceptional

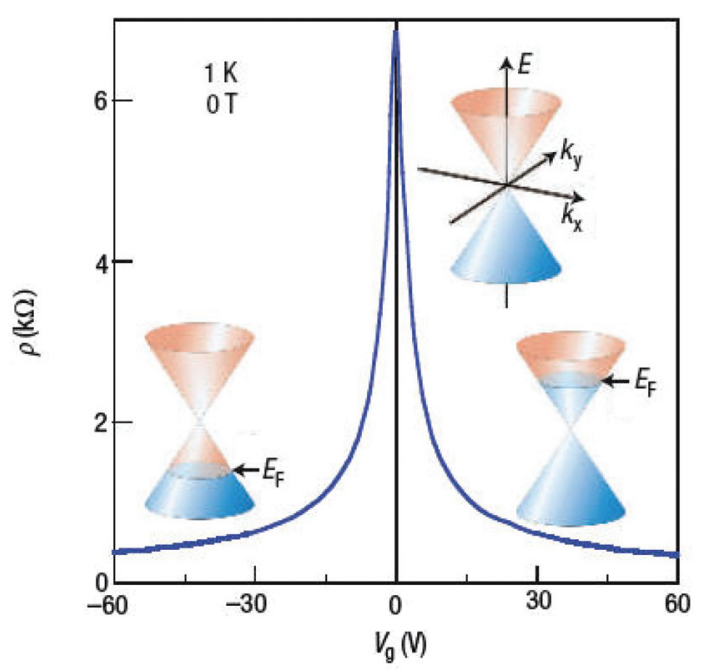

Fig. 2 Ambipolar electric field effect in single-layer graphene. The insets show its low-energy spectrum, indicating changes in the position of the Fermi energy $E_{\mathrm{F}}$ with varying gate voltage $V_{\mathrm{g}}$. (Taken from ref. 4. Reproduced with permission from Nature Publishing Group.) carrier mobility, graphene is highly applicable to RF electronics. Hence, some of the earliest studies on graphene electronics were focused on high-speed transistors and unique analog circuitry exploiting ambipolarity. ${ }^{34,38,46,48,59,83}$

\section{Electronic properties of layered TMDs}

One aspect of graphene that severely limits its applicability is the lack of bandgap and the resulting leakage of current during transistor operation. ${ }^{8}$ Various functionalized forms of graphene can introduce an energy gap but only at the cost of reduced electronic properties, such as degraded mobility. ${ }^{84,85}$ Several TMDs, on the other hand, are gapped semiconductors $^{65}$ and still retain the exotic qualities because their thicknesses are comparable to that of graphene (graphene is roughly $\sim 3 \AA^{86}$ thick while most monolayer TMDs are $\sim 7 \AA$ thick ${ }^{2,87}$ ). For example, the bandgap of a monolayer $\mathrm{MoS}_{2}$ is found to be around $1.9 \mathrm{eV}$ with a thickness of $\sim 6.9 \AA^{2,25}$

Generally, TMDs have the stoichiometric formula of $\mathrm{MX}_{2}$, where $\mathrm{M}$ is a transition metal from group 4 to 10 , and $\mathrm{X}$ is a chalcogen ( $\mathrm{S}, \mathrm{Se}, \mathrm{Te})$. The crystalline structure consists of transition metal atoms forming hexagonal crystal lattices that reside between chalcogen atom layers. Although most TMDs based on group 4-7 elements are layered (such as $\mathrm{MoSe}_{2}, \mathrm{WS}_{2}$, $\mathrm{WSe}_{2}, \mathrm{HfSe}_{2}, \mathrm{NbSe}_{2}, \mathrm{TaSe}_{2}, \mathrm{NiTe}_{2}$, etc.), it is worth noting that some TMDs based on group 8-10 elements (such as $\mathrm{FeSe}_{2}$ ) are not layered materials. ${ }^{7}$

Fundamentally, all 2D materials are inevitably affected by the interlayer coupling and the quantum confinement. ${ }^{7,66}$ Layered multilayers exhibit dramatically different electronic properties from 2D monolayers. ${ }^{7,65,66}$ The band structure of these materials can be calculated from the density functional theory. ${ }^{65,66}$ Fig. 3 depicts the band structure obtained for bulk, quad-, bi-, and mono-layer of an $\mathrm{MoS}_{2}$. With reduced layer thickness, the indirect bandgap becomes larger, while the direct excitonic transition barely changes. The lowest energy transition changes as the black arrow indicates, and this

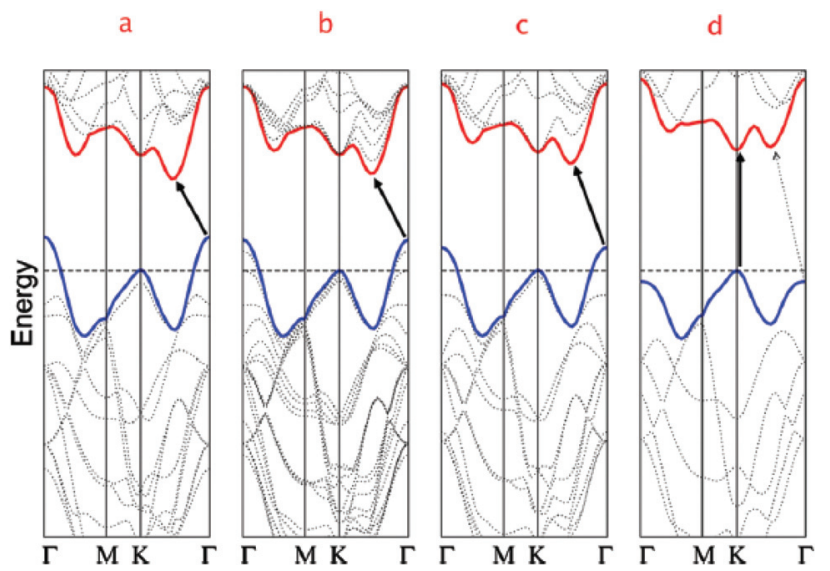

Fig. 3 Calculated band structures of (a) bulk $\mathrm{MoS}_{2}$, (b) quadlayer $\mathrm{MoS}_{2}$, (c) bilayer $\mathrm{MoS}_{2}$, and (d) monolayer $\mathrm{MoS}_{2}$. The solid arrows indicate the lowest energy transitions. (Taken from ref. 66. Reproduced with permission from the American Chemical Society.) 
results in an indirect to direct bandgap transition. This, in turn, also results in a larger bandgap for monolayer $\mathrm{MoS}_{2}$. Experimentally, this development is confirmed as enhanced photoluminescence in single-layered $\operatorname{MoS}_{2}{ }^{66}$ This property is a direct consequence of quantum confinement and can be observed in other 2D TMDs, such as $\mathrm{MoSe}_{2}, \mathrm{WS}_{2}$, and WSe $_{2}{ }^{1,66,83,88,89}$ Consequently, semiconducting TMD monolayers tend to have larger bandgaps than their bulk counterparts.

Fig. 4 shows the calculated band alignments for several known monolayer TMDs. ${ }^{65} \mathrm{~A}$ few notable trends can be observed from the band alignments. As the atomic number of the $\mathrm{X}$ (chalcogen) increases, both the valance band maximum and the conduction band minimums increase. Also, the valance band offset tends to be larger than the conduction band offset, leading to smaller bandgaps for TMDs with larger atomic number chalcogens. Moreover, the conduction and valence band of W-based TMDs are higher than those of Mo-based TMDs. The band offsets are generally determined by the repulsion strength from the cation d orbitals and the anion p orbitals. ${ }^{66}$ The overlap integral of $d$ and p orbitals and the differences in energy as well as the $\mathrm{M}-\mathrm{X}$ bond length determine the overall band offsets and its alignment. ${ }^{66}$

An interesting aspect of the $2 \mathrm{D}$ material is that some materials (e.g. graphene, $\mathrm{WSe}_{2}$ ) tend to be p-type while some materials (e.g. $\mathrm{MoS}_{2}, \mathrm{MoSe}_{2}$ ) tend to be n-type in ambient air. Atomically thin $\mathrm{p}-\mathrm{n}$ junctions have been formed exploiting this trait. ${ }^{91}$ Furthermore, physisorption of gas adsorbates either depletes or accumulates charges resulting in opposite effects for n-type and p-type 2D materials. For example, physisorbed $\mathrm{O}_{2}$ and $\mathrm{H}_{2} \mathrm{O}$ molecules deplete n-type $\mathrm{MoS}_{2}$, which weakens the electrostatic screening and leads to the enhancement of photoluminescence. ${ }^{92}$ In $\mathrm{WSe}_{2}$, the opposite effect was observed. ${ }^{92}$

Finally, it is worth noting that there are two possible polymorphs of monolayer TMDs (Fig. 5). For a trigonal prismatic $(2 \mathrm{H})$ phase, atoms of each chalcogen layer are directly on top

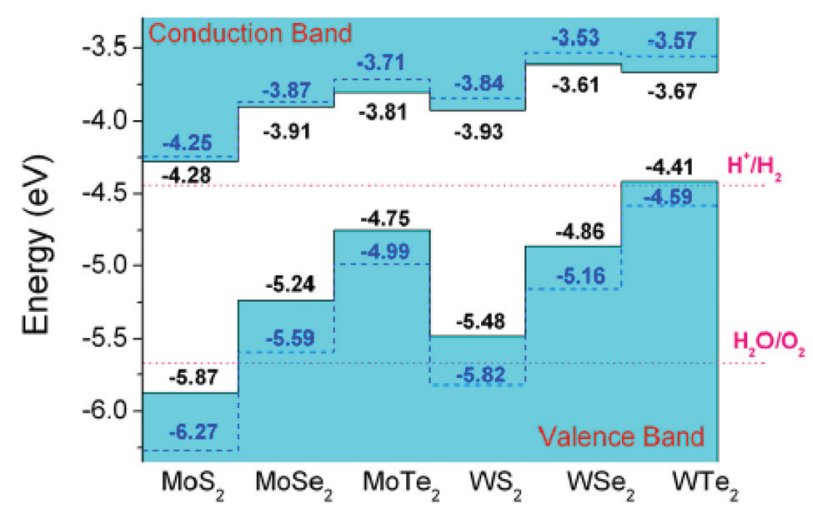

Fig. 4 Calculated band alignment for transition metal dichalcogenide monolayers. Solid lines and dashed lines are obtained by different simulation approaches. The dotted lines indicate the water reduction $\left(\mathrm{H}^{+} / \mathrm{H}_{2}\right)$ and oxidation $\left(\mathrm{H}_{2} \mathrm{O} / \mathrm{O}_{2}\right)$ potentials. The vacuum level is taken as zero reference. (Taken from ref. 65 . Reproduced with permission from the American Institute of Physics.)
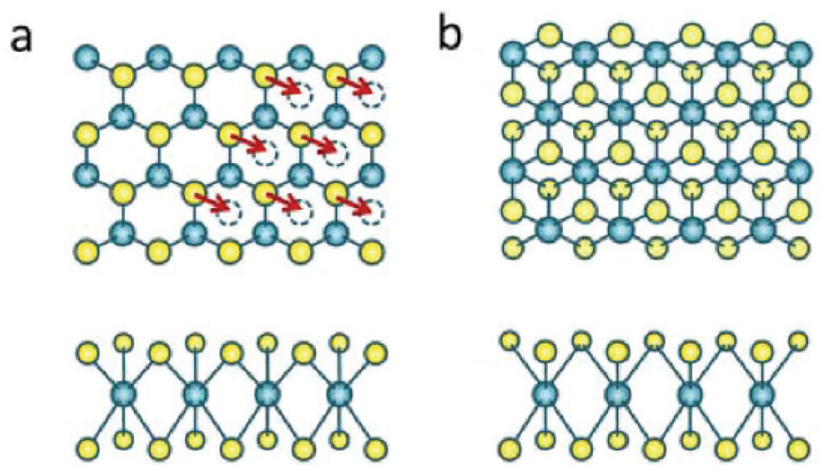

Fig. 5 Illustration of the $\mathrm{MoS}_{2}$ structure viewed from the out-of-plane and in-plane axes. The blue and yellow spheres represent Mo and $\mathrm{S}$ atoms. (a) $2 \mathrm{H}$ phase $\mathrm{MoS}_{2}$. Only the $\mathrm{S}$ atoms on the upper planes can be seen from the $c$-axis as those on the lower planes are directly underneath the top atom. The arrows in panel a show the local S plane glide motion that leads to local transformation to $1 \mathrm{~T}$ structure. (b) $1 \mathrm{~T}$ phases. (Taken from ref. 90. Reproduced with permission from the American Chemical Society.)

of the other chalcogen layers. The trigonal antiprismatic phase (often referred as octahedral or 1T), on the other hand, has alternating chalcogen layers offset from each other as shown in Fig. $5 \mathrm{~b} .{ }^{90}$ This difference in the atomic arrangement has a profound effect on the band structure. Progressive filling of the non-bonding $\mathrm{d}$ bands of the transition metal atoms leads to either metallic or semiconducting behaviour. For $\mathrm{MoS}_{2}$, the $2 \mathrm{H}$ form is semiconducting while the $1 \mathrm{~T}$ form is metallic. ${ }^{90}$ The preferred phase of a TMD is mainly determined by the number of d-electrons in the transition metal. For example, group 4 TMDs are mostly trigonal antiprismatic while group 6 TMDs are predominantly trigonal prismatic. Group 5 TMDs are found in both forms. ${ }^{7}$

\section{Graphene-based analog/RF circuits}

The lack of bandgap in graphene limits graphene's role in traditional logic applications. However, this requirement of a high on/off ratio is less stringent in analog/RF electronics. ${ }^{8}$ In a single-stage amplifier, for example, the transistor operates in the on-state while a small signal is superimposed on the DC gate voltage. Furthermore, the charge carriers in graphene exhibit extremely high carrier mobility with zero effective mass. ${ }^{10,77,86}$ Combined with its unique two dimensional structure, graphene is potentially well suited for high speed RF applications. Transistors with cut-off frequencies as high as $300 \mathrm{GHz}$ were demonstrated with both exfoliated ${ }^{30}$ and CVD synthesized $^{42}$ graphenes. Hence, the unique properties of graphene have led to plenty of novel RF components, including mixers, ${ }^{38-40}$ frequency multipliers, ${ }^{34,36,46}$ modulators, ${ }^{35,37}$ and amplifiers. ${ }^{41-43}$

\section{Frequency multipliers}

Frequency multiplication has always been an integral part of various analog electronics and communication systems. Most 
(a)

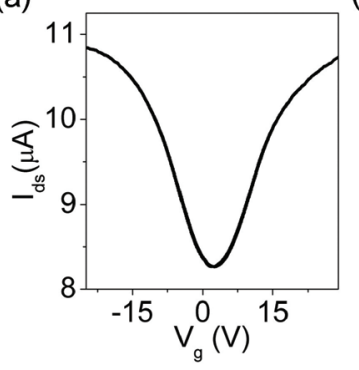

(b)

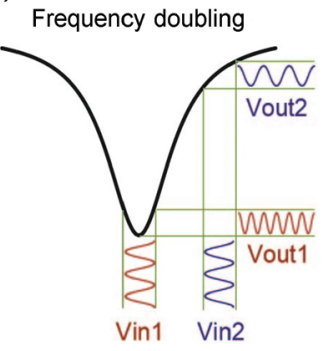

Fig. 6 (a) Typical $I_{d}-V_{g}$ curve of a graphene transistor. (b) Frequency modulation is achieved by interchanging the bias point from a region dominated by electron (or hole) carriers to the charge neutrality point. (Taken from ref. 37. Reproduced by permission of Nature Publishing Group.)

frequency multipliers, however, are realized by generating harmonics from nonlinear components such as diodes and filtering out the necessary signals with additional components. ${ }^{93}$ The major drawback of this method is the low efficiency $(<30 \%)$ and the requirement of additional filtering components. With graphene-based transistors, however, it is possible to multiply signals very efficiently with only one transistor by exploiting the ambipolar transport behaviour. ${ }^{34,35}$

As shown in Fig. 6a, positive gate voltage induces electrons with NFET-type response while negative gate voltage induces holes with PFET-type response. The gate voltage that results in minimum conductance is called the Dirac point voltage or the charge neutrality point (CNP) voltage. The Dirac point voltage may drift from $0 \mathrm{~V}$ depending on the environmental doping effects. ${ }^{18}$ Nevertheless, the environmental doping can be controlled with meticulous sample preparation and a capping passivation layer. ${ }^{77}$

As shown in Fig. 6b, applying an oscillating input signal $\left(V_{\text {in2 }}\right)$ on either side of the Dirac point voltage will result in an output signal $\left(V_{\text {out2 }}\right)$ with the same frequency. However, when the input signal $\left(V_{\text {in1 }}\right)$ is directly applied at the Dirac point voltage, the frequency of the output signal $\left(V_{\text {out1 }}\right)$ will be twice the original value. This is the result of a sudden change in the phase of the signal when the original input signal crosses the Dirac point.

Several frequency multipliers, including doublers using one transistor (Fig. 7) and a tripler using two transistors (Fig. 8), have been demonstrated. The tripler uses two graphene transistors with different Dirac point voltages to obtain two conduction dips for additional change in the phase. Remarkably, the output signals of these multipliers have very high spectral purity with over $90 \%$ of the RF power in the multiplied frequency range. ${ }^{34,46}$ It is important to note that rather complex circuits with multiple transistors/diodes and other passive components are required to enable frequency multiplication with current CMOS techno$\operatorname{logy} .{ }^{93}$ The ambipolarity is also the key aspect of modulators and mixers based on graphene transistors.

\section{Modulators}

In today's communication systems, modulation is an essential function that embeds the baseband signal (information) into a

(a)

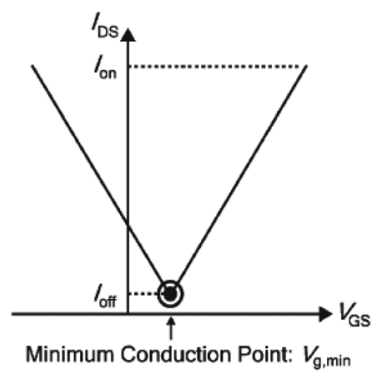

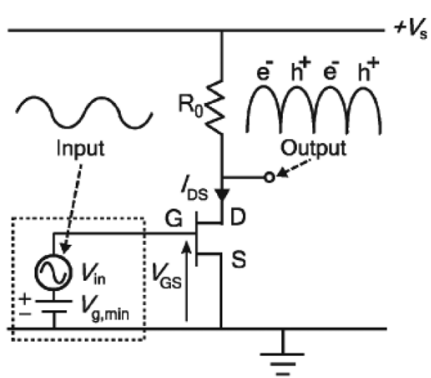

(b)
Fig. 7 (a) Linear approximation of the transfer characteristic of ambipolar graphene FETs. The minimum conduction point is equal to the quiescent bias point for full-wave rectification and frequency doubling. (b) With the device being biased at the minimum conduction point, electrons and holes conduct alternatively in half-cycles of the output signal. (Taken from ref. 34. Reproduced with permission from the Institute of Electrical and Electronics Engineers (IEEE).)

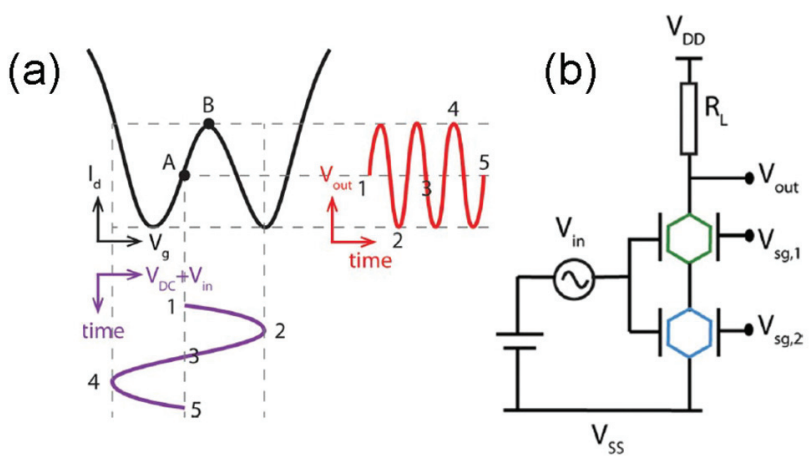

Fig. 8 (a) Schematic diagram illustrating the operation principle of a graphene frequency tripler. The input waveform and output waveform are shown in purple and red respectively. The input dc voltage bias (point $A$ ) is chosen halfway between the voltages where minimum current occurs and the central current peak (point B). Points in time 1 through 5 in the input cycle correspond to those labelled in the output waveform. (b) Circuit schematic of the graphene frequency tripler. $V_{\text {sg1 }}$ and $V_{\text {sg2 }}$ are dc voltages used to control the threshold voltage separation electrostatically between two graphene FETs. (Taken from ref. 46. Reproduced with permission from the American Chemical Society.)

carrier wave so that it can be successfully broadcasted through a medium such as air. The basic modulation techniques map the information by varying up to three different parameters (amplitude, frequency and phase) of the carrier wave to represent the data. The most fundamental modulation techniques that correspond to each of these three parameters are amplitude modulation (AM), frequency modulation (FM), and phase modulation (PM). ${ }^{94}$

Similar to frequency multiplication as explained in the previous section, the unique ambipolar gate response of graphene transistors allows simple implementation of the three basic modulation schemes as illustrated in Fig. 9. The amplitude, frequency, and phase of the output voltage are determined by the operating gate bias point of the graphene transistor. For example, amplitude modulation (AM) can be achieved by utilizing the transconductance change over the gate voltage 


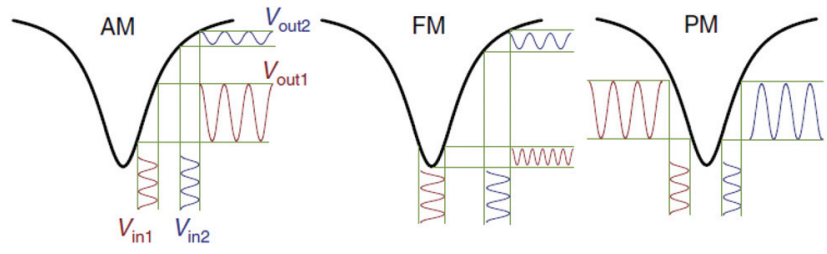

Fig. 9 Illustrations of amplitude, frequency and phase modulation of a sinusoidal wave achieved by operating a single ambipolar graphene transistor at different gate biases. (Taken from ref. 37. Reproduced with permission from Nature Publishing Group.)

difference. Frequency modulation (FM) is achieved by interchanging the bias point from a region dominated by electron (or hole) carriers to the charge neutrality point. Similarly, phase modulation (PM) is realized by changing the bias point from a region dominated by an electron (or hole) carrier to a region dominated by the hole (or electron) carrier. The most basic modulation schemes that encode 1 bit of data (i.e. 0 or 1 ) per signal are called binary modulation schemes. Several pioneering studies have been done to verify the fully functioning binary modulators. ${ }^{35,95}$

By combining two or more of these binary modulation schemes, it is possible to extend this technique into quater- nary modulation schemes such as quadrature phase-shift keying (QPSK). ${ }^{37}$ Specifically, QPSK is the key building unit for highly efficient modulation techniques that are widely used in today's telecommunication standards.

Fig. 10a shows a typical QPSK transmitter structure used in modern digital communication. A binary data stream is demultiplexed into the in-phase component (I) and the quadrature-phase component (Q). I and Q components are encoded onto two orthogonal basis functions, such as a sine wave and a cosine wave, respectively, before they are summed to generate a QPSK modulated signal.

In this work, two transistors are used to demonstrate the QPSK modulation (Fig. 10b). Actual microscope images under a blue filter were overlaid on top of the circuit diagram. A sinusoidal wave from the function generator was connected to a simple off-chip resistance-capacitance-capacitance-resistance (RC-CR) phase shift network to generate two orthogonal wave functions with $90^{\circ}$ phase difference. The sinusoidal input is shifted by $+45^{\circ}$ in the $\mathrm{CR}$ branch and by $-45^{\circ}$ in the RC branch. Then each of these signals is summed internally by the function generators with two square waves (Idata and Qdata) and fed to the gates of each transistor. The outputs (VI and VQ) are then summed to generate the final QPSK modulated signals. These signal components are plotted in Fig. 10c. (a)

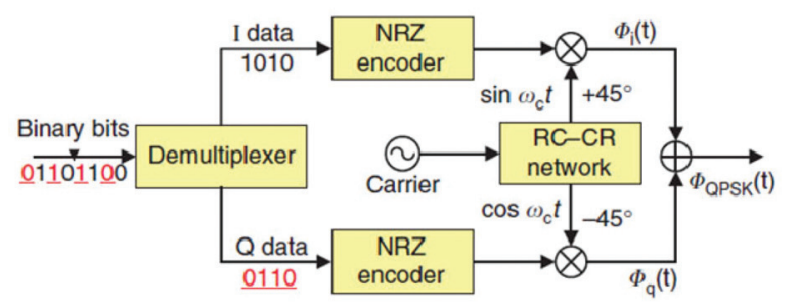

(b)

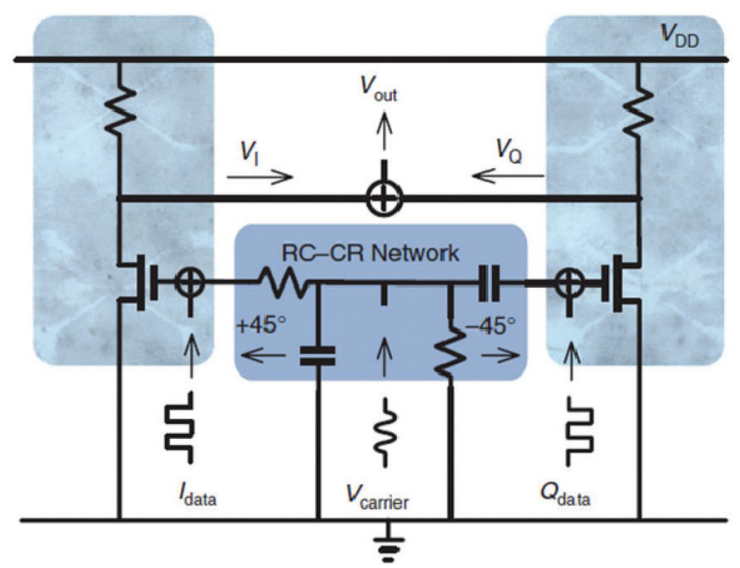

(c)

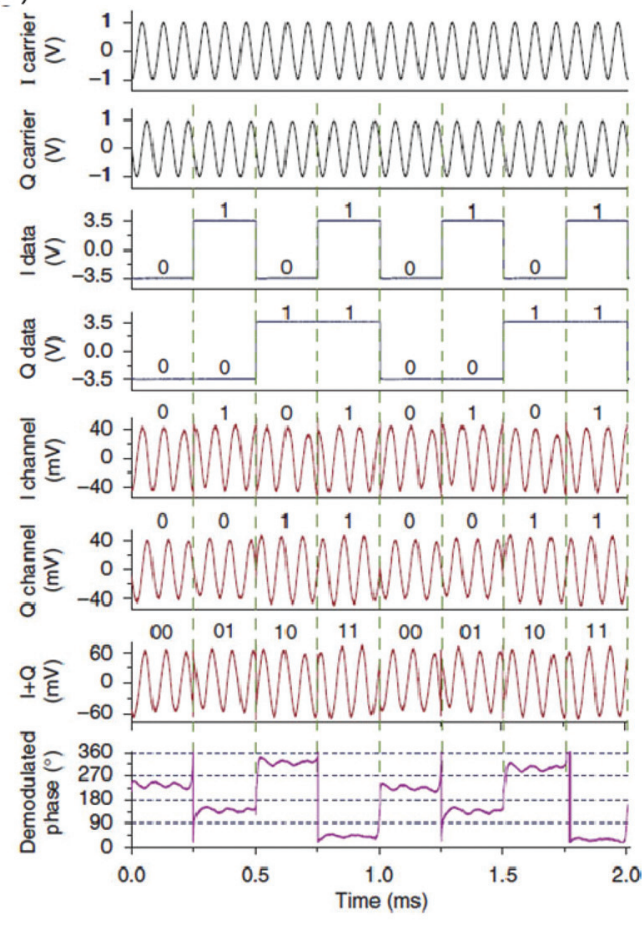

Fig. 10 QPSK demonstrated with two all-graphene transistors. (a) A conceptual diagram of a conventional QPSK transmitter structure. The NRZ encoder is a non-return-to-zero encoder, where 1 is represented by a positive voltage state and 0 is represented by a negative voltage state. The RC-CR network is the resistance-capacitance-capacitance-resistance phase shift network that generates two orthogonal wave functions with $90^{\circ}$ phase difference. (b) An all-graphene circuit diagram of the QPSK system using two transistors. The actual microscopic image of the all-graphene circuit under a blue filter is shown. (c) Time domain plots of the input and output signals demonstrating QPSK modulation scheme. (Taken from ref. 37. Reproduced with permission from Nature Publishing Group.) 
The Icarrier and the Qcarrier are the orthogonal carrier signals. The data bitstream with $00,01,10,11$ is represented by the in-phase component Idata and the quadrature-phase component Qdata as shown in the plot. Modulating Icarrier with Idata results in phase changes in Ichannel and the same applies to Qcarrier, Qdata, and Qchannel. Data bit 0 and 1 in Idata correspond to phase of $180^{\circ}$ and $0^{\circ}$ in Ichannel. Similarly, Data bit 0 and 1 of Qdata correspond to phase of $90^{\circ}$ and $270^{\circ}$ in Qchannel. The sum of Ichannel and Qchannel is the final output signal $(\mathrm{I}+\mathrm{Q})$, which has distinct phase shifts of $225^{\circ}, 135^{\circ}, 315^{\circ}$, and $45^{\circ}$, each corresponding to binary data of $00,01,10$, and 11 , respectively. To validate the result, the instantaneous phase information was extracted from the final output signal $(\mathrm{I}+\mathrm{Q})$ and plotted as a demodulated phase (Fig. 10c, bottom panel).

\section{Mixers}

Another inevitable consequence of ambipolarity is an acute nonlinearity in the vicinity of the Dirac voltage. The transfer characteristics of an ambipolar graphene FET are symmetrical around the Dirac voltage. This symmetrical characteristic usually results in a very significant quadratic component, and therefore mixers based on this characteristic can effectively suppress odd order intermodulation and lead to lower spurious emissions in the circuit. ${ }^{38}$ A mixer based on graphene was demonstrated at a frequency of $10 \mathrm{MHz}$ using graphene grown by chemical vapour deposition, and a high-quality factor was observed with a third-order intercept point of $+13.8 \mathrm{dBm} .{ }^{38}$

A high-performance mixer fabricated by integrating graphene transistors and passive components on a single silicon carbide wafer (Fig. 11) was also developed. ${ }^{39}$ The importance of this work is twofold: demonstration of multi-level, waferscale integration, and verification of superior thermal stability in graphene-based transistors compared to conventional ones. The absence of strong temperature dependence in graphene is attributed to a degenerate doping level in graphene and a nearly temperature-independent scattering mechanism associated with optical phonons at high biases. ${ }^{39}$ The mixer operated at frequencies up to 10 gigahertz and exhibited exceptional thermal stability with little reduction in performance up to 400 kelvin.

In contrast to the previous two studies on fundamental mixers, a subharmonic graphene FET mixer (Fig. 12) was recently demonstrated. ${ }^{40}$ A subharmonic mixer needs only half the local oscillator (LO) frequency of a fundamental mixer. In addition, subharmonic mixers suppress the LO noise, and the wide frequency gap between the RF and LO signals simplifies the LO and RF separation. Also, a balun is not required, as opposed to conventional subharmonic resistive FET mixers, which require two FETs in parallel and a balun for feeding the two out-of-phase LO signals. ${ }^{40}$ The circuit topology is shown in Fig. 12. Modulating the gate voltage results in a time-varying channel resistance $R_{\mathrm{ds}}(t)$. Due to the biasing at the Dirac point, $R_{\mathrm{ds}}(t)$ varies as a function of $2 \times f_{\mathrm{LO}}$ (frequency doubling). The time-varying reflection coefficient is $\Gamma(t)=\left(R_{\mathrm{ds}}(t)-\right.$ $\left.Z_{\mathrm{o}}\right) /\left(R_{\mathrm{ds}}(t)+Z_{\mathrm{o}}\right)$ seen in the drain of the graphene FET, where
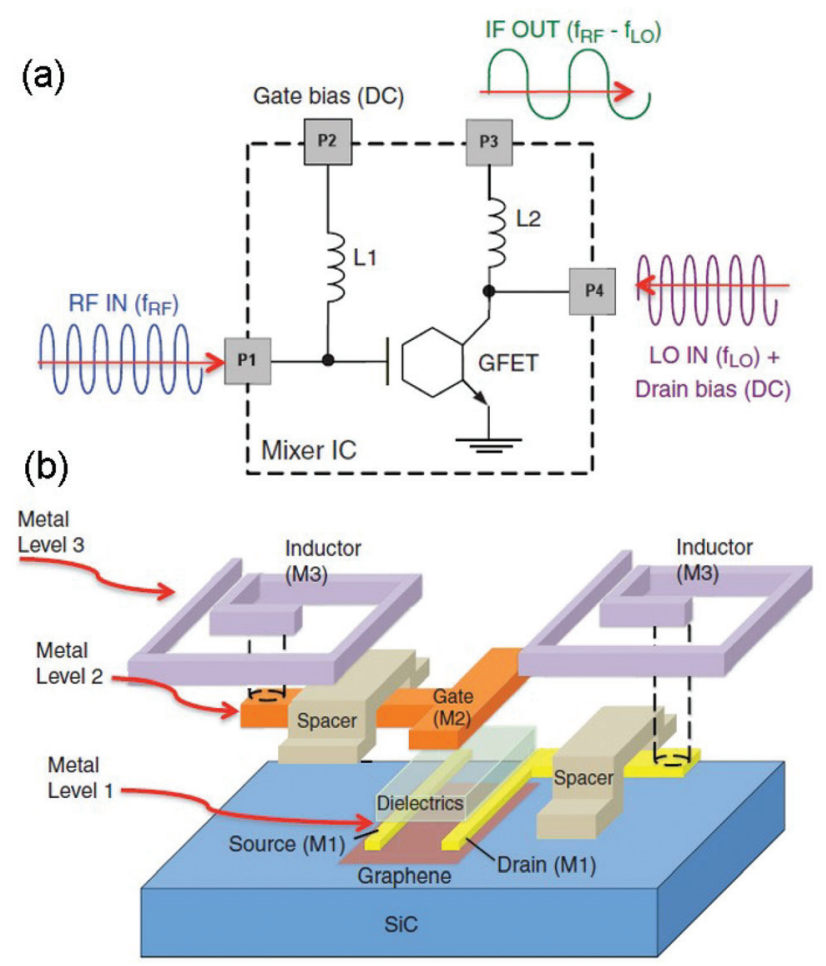

Fig. 11 (a) Circuit diagram of a graphene RF frequency mixer on a SiC wafer. (b) Illustration of a graphene mixer circuit. The design components include a graphene transistor and two inductors connected to the gate and the drain of the GFET. Three distinct metal layers of the graphene IC are represented by M1, M2, and M3. (Taken from ref. 39. Reproduced with permission from the American Association for the Advancement of Science.)

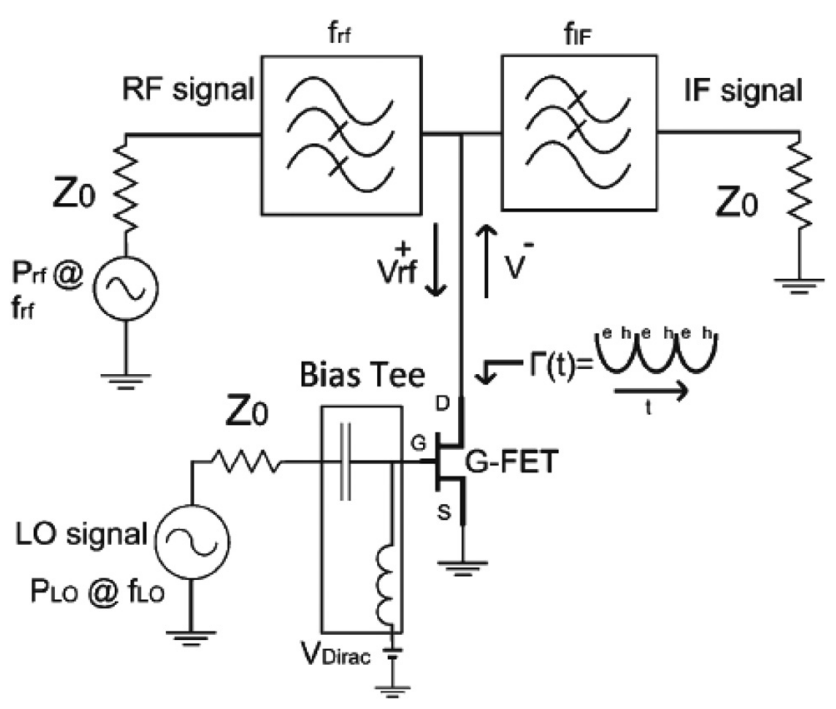

Fig. 12 Circuit structure of the subharmonic resistive graphene FETbased mixer. (Taken from ref. 40. Reproduced with permission from the Institute of Electrical and Electronics Engineers (IEEE).)

$Z_{\mathrm{o}}=50 \Omega$ is the system impedance. By feeding an RF signal $V_{\mathrm{RF}}{ }^{+}(t)$ to $\Gamma(t)$, the reflected signal (i.e. output voltage) $V^{-}(t)=$ $\Gamma(t) V_{\mathrm{RF}}^{+}(t)$ is generated. The reflected voltage has frequency 
components at $\left|f_{\mathrm{RF}} \pm 2 n f_{\mathrm{LO}}\right|$, and the IF component $\left(f_{\mathrm{IF}}=\mid f_{\mathrm{RF}}\right.$ $\left.-2 f_{\mathrm{LO}} \mid\right)$ is the desired one for the down-conversion mixer. A down-conversion loss of $24 \mathrm{~dB}$ was obtained with $f_{\mathrm{RF}}=2 \mathrm{GHz}$, $f_{\mathrm{LO}}=1.01 \mathrm{GHz}$, and $f_{\mathrm{IF}}=20 \mathrm{MHz}$.

\section{Amplifiers}

Before we continue on to amplifiers, different terms related to different gains have to be clarified. In this paper, $f_{\mathrm{T}} \approx$ $g_{\mathrm{m}} / 2 \pi C_{\mathrm{ox}}$ denotes the cut-off frequency, defined as the frequency at which the current gain becomes unity $\left(g_{\mathrm{m}}\right.$ is the transconductance and $C_{\mathrm{ox}}$ is the gate capacitance). $f_{\mathrm{T}}$ is obtained from the short-circuit current gain $h_{21}$, while the load impedance is nonzero. $f_{\max }$ is related to the power gain obtained assuming the best impedance matching. It is the highest possible operating frequency before a transistor loses its ability to amplify power. Open-circuit voltage gain refers to the condition of infinitely high load impedance. The opencircuit voltage gain is related to the intrinsic gain $G_{\text {int }}$, obtained from the ratio $g_{\mathrm{m}} / g_{\mathrm{d}}$, where $g_{\mathrm{m}}$ is the transconductance and $g_{\mathrm{d}}$ is the output conductance.

It is very important to distinguish these figures of merit for graphene transistors because of graphene's weak saturation behaviour. ${ }^{8}$ High $f_{\mathrm{T}}$ numbers reported for graphene transistors can be misleading. This $f_{\mathrm{T}}$ results directly from graphene's high mobility. ${ }^{8}$ However, since the drain current does not saturate in these devices, they exhibit limited power gain and voltage gain than what is required for practical RF circuit applications. ${ }^{8,42,43}$

Hence, several studies were focused on obtaining sizeable voltage gain in graphene transistors. ${ }^{41-43}$ Han et al. partially solved the drain current saturation problem in graphene FET with an extremely thin and planar gate dielectric. ${ }^{43}$ They have exploited the fact that the shift of the Dirac point with good gate control should be equal to half of the applied drain bias. ${ }^{49}$ This shifting of the Dirac point translates into a clear drain current saturation. However, graphene is a highly inert material and techniques such as atomic layer deposition are not ideal methods to form a pinhole-free dielectric. Hence, they used a chemical-mechanical polishing (CMP) method to form a very flat and thin bottom gate dielectric $\left(\mathrm{HfO}_{2}, 4 \mathrm{~nm}\right.$ thick) underneath the graphene layer. The graphene commonsource amplifier fabricated with this method exhibited $5 \mathrm{~dB}$ low frequency gain with the $3 \mathrm{~dB}$ bandwidth greater than 6 $\mathrm{GHz}^{43}$

There are other studies that also exploited the Dirac voltage shifting effect. ${ }^{41}$ In Fig. 13, a voltage amplifier was realized with a complementary push-pull configuration. By applying the supply voltage $V_{\mathrm{DD}}$, the potential of the graphene channel in G2 increases with respect to that of G1, which therefore shifts the Dirac point of G2 to higher input voltages. Complementary operation is obtained between the Dirac points of the two FETs, where the increase in input voltage $V_{\text {IN }}$ causes the resistance of G2 to increase and the resistance of G1 to decrease. This results in a large rate of increase for the output voltage $V_{\text {OUT }}$ as the input voltage $V_{\text {IN }}$ changes, and doubles the voltage gain $A_{\mathrm{v}}$ compared to a single graphene FET-based

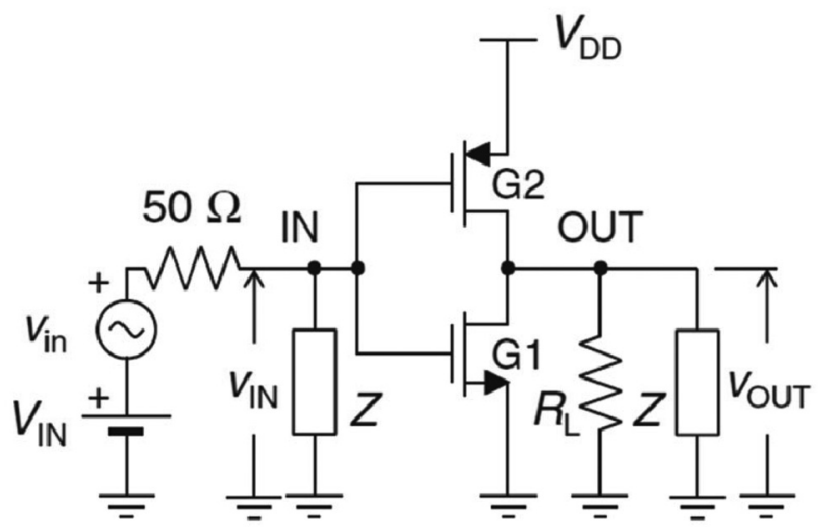

Fig. 13 A circuit diagram of the graphene transistor-based amplifier with complementary push-pull configuration. $Z=1 \mathrm{M} \Omega$ is the input impedance of the oscilloscope while $50 \Omega$ is the output resistance of the input voltage source. $V_{I N}$ is the DC bias voltage and $v_{\text {in }}(t)$ is the AC component of the input signal. The amplifier is additionally loaded with $R_{\mathrm{L}}$ to simulate the next amplifying stage. (Taken from ref. 41. Reproduced with permission form Wiley.)

common-source amplifier. The amplifier had a voltage gain of $3.7(11.4 \mathrm{~dB})$ at $10 \mathrm{kHz}$, a total harmonic distortion in the audio frequency range of $<1 \%$, a unity-gain frequency of 360 $\mathrm{kHz}$ and $\mathrm{a}-3 \mathrm{~dB}$ bandwidth of $70 \mathrm{kHz} .{ }^{41}$

Subsequently, state-of-the-art graphene FET-based amplifiers with intrinsic cut-off frequencies above $300 \mathrm{GHz}$ were also developed. ${ }^{42}$ This work was done in wafer-scale with both CVD-synthesized and epitaxially grown graphene, and the value of $f_{\mathrm{T}}$ is the current record for graphene-based RF electronics. These devices also exhibited voltage and power gains reaching $20 \mathrm{~dB}$. This was achieved by minimizing the scattering effect at the interfaces with ultra-smooth, diamond-like carbon substrates. ${ }^{29}$ The channel doping was also minimized to greatly improve the transconductance and current saturation. Fig. 14a and b show interesting results from these devices. $f_{\mathrm{T}}$ for both CVD graphene and epitaxial graphene exhibits the typical $1 / L$ trend (Fig. 14a). There is very little performance gap between CVD graphene and epitaxial graphene. $f_{\max }$, on the other hand, reaches a peak value at around $200 \mathrm{~nm}$ channel length (Fig. 14b). This peak in $f_{\max }$ is the result of competing contributions from $f_{\mathrm{T}}$, gate resistance, and output conductance $g_{\mathrm{d}}$ as the gate length decreases. ${ }^{94,95}$ Since the $f_{\max }$ value does not represent the intrinsic performance, but is affected by extrinsic components, the power gain can be improved by better saturation behaviour and the optimization of parasitic components.

\section{CMOS/graphene integrated}

Since the discovery of graphene, defining its role in the current CMOS-dominated semiconductor industry was the main goal of many researchers. Due to its unique advantages and the ease of transfer, many believe it will have several key applications tightly integrated with CMOS technology. ${ }^{44,47,96}$ As shown previously, several RF electronic components have been integrated in wafer-scale circuitry. With significant 

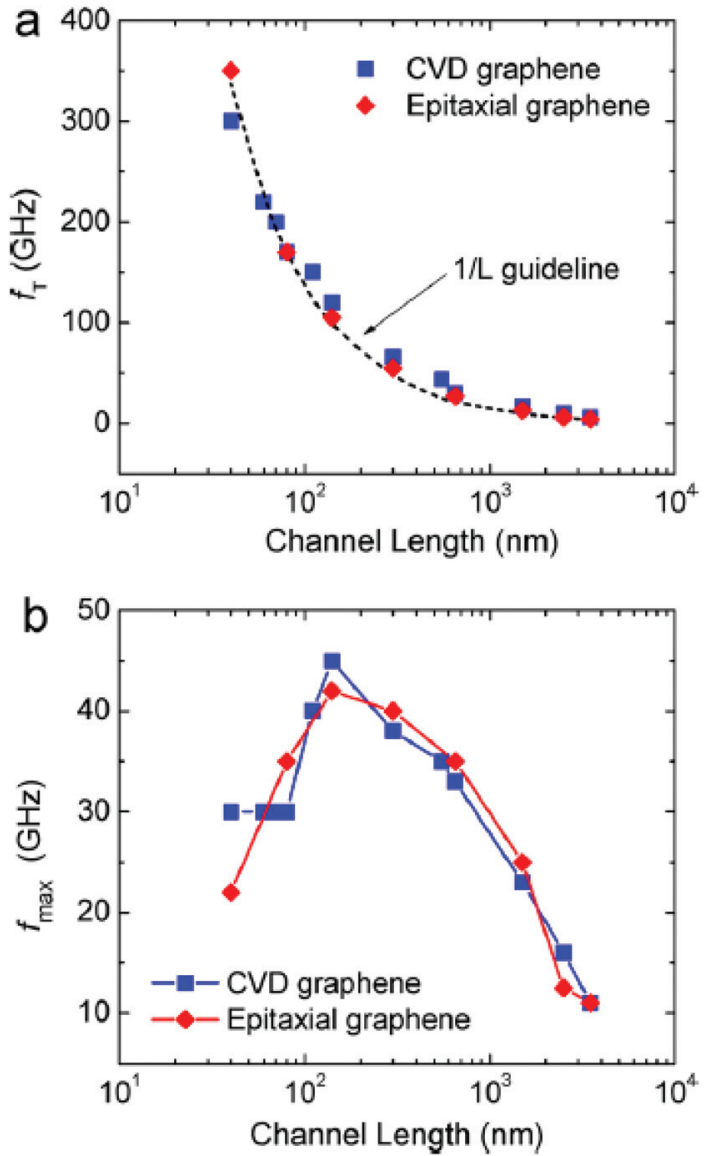

Fig. 14 (a) Scaling behaviour of $f_{\mathrm{T}}$ versus channel length, showing the clear $1 / L$ dependence. (b) $f_{\max }$ versus channel length with the peak $f_{\max }$ obtained at a channel length of $140 \mathrm{~nm}$. (Taken from ref. 42. Reproduced with permission from the American Chemical Society.)

improvement in fabrication technology, graphene components can now be integrated into circuitry with higher complexity and system functionality. A multi-stage graphene RF receiver integrated circuit is shown (Fig. 15). ${ }^{44}$ One significant improvement in this work is the preservation of graphene quality during fabrication by applying a passive-first, active-last integration scheme (Fig. 15a). This way, graphene FETs are not exposed during the downstream back-end-of-line (BEOL) process, hence, preventing the degradation of the graphene quality. The entire fabrication flow does not require process steps higher than $400{ }^{\circ} \mathrm{C}$.

The circuit operates as an $\mathrm{RF}$ receiver with front-end performing signal amplification, filtering, and down conversion mixing. All the components are fully integrated into a $0.6 \mathrm{~mm}^{2}$ area and fabricated in $200 \mathrm{~mm} \mathrm{Si}$ fab, showing complete silicon CMOS process compatibility. The first two stages are designed as bandpass amplifiers, and the third stage performs mixing with the local oscillator (LO) signal which down-converts the GHz RF signal to intermediate frequency (IF) in the $\mathrm{MHz}$ range (Fig. 15b). A fundamental drain-pumped mixer design is employed since it leads to better performance based on the characteristics of graphene-based FETs, and results in (a)
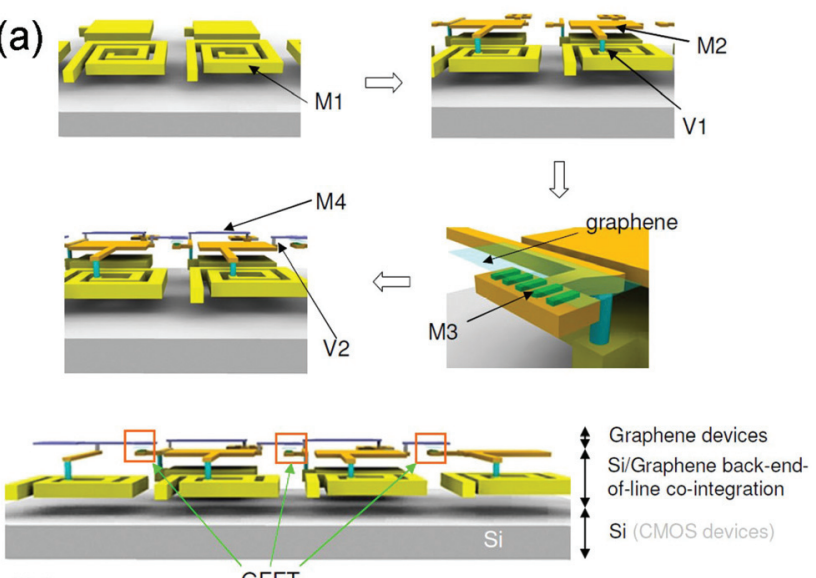

(b)

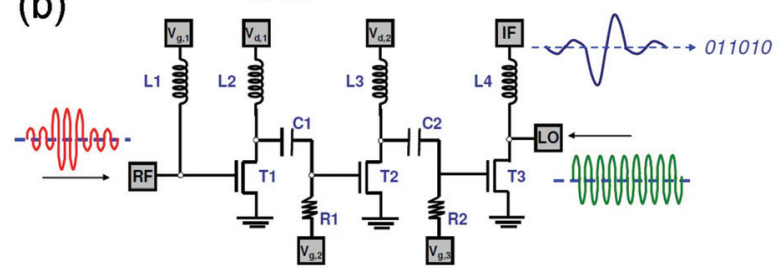

Fig. 15 (a) Schematic of the passive-first active-last process flow showing integrated IC fabrication with standard Si BEOL process. Four metal levels (M1-M4) were required. (b) Circuit schematic of graphene $\mathrm{RF}$ receiver. Inductor $\mathrm{L} 4$, connected at the drain of mixing graphene FET, serves two purposes: it resonates the drain capacitance of transistor T3 to provide a large LO voltage swing at the drain terminal, and also attenuates the LO signal leakage toward the IF port. (Taken from ref. 44. Reproduced with permission from Nature Publishing Group.)

low LO power requirements $(<0 \mathrm{dBm})$. In Fig. 15b, inductor L4 serves two purposes: It resonates the drain capacitance of transistor T3 to provide a large LO voltage swing at the drain terminal, and also attenuates the LO signal leakage toward the IF port.

To demonstrate the functionality of the graphene IC, an RF carrier of $4.3 \mathrm{GHz}$ was amplitude-modulated with a bitstream and sent to the receiver (Fig. 16). Measured single-bit waveforms in Fig. 16a show IF output signals from the receiver. In Fig. 16b, the bitstream composed of ASCII code modulated at $20 \mathrm{Mb} \mathrm{s}^{-1}$ was received and demodulated. The original bitstream comprising 3 letters ( 24 bits) was recovered by the graphene receiver with low distortion.

\section{Graphene-based digital circuits}

Even with the inherent lack of bandgap, there were several efforts utilizing graphene in digital electronics by exploiting graphene's unique properties.

\section{Inverters}

Pioneering work on graphene-based inverters was achieved by fabricating complementary-like logic inverters based on graphene field-effect transistors (Fig. 17a). ${ }^{49}$ The complementary p- and n-type FET pairs were fulfilled through superposition of 
(a)

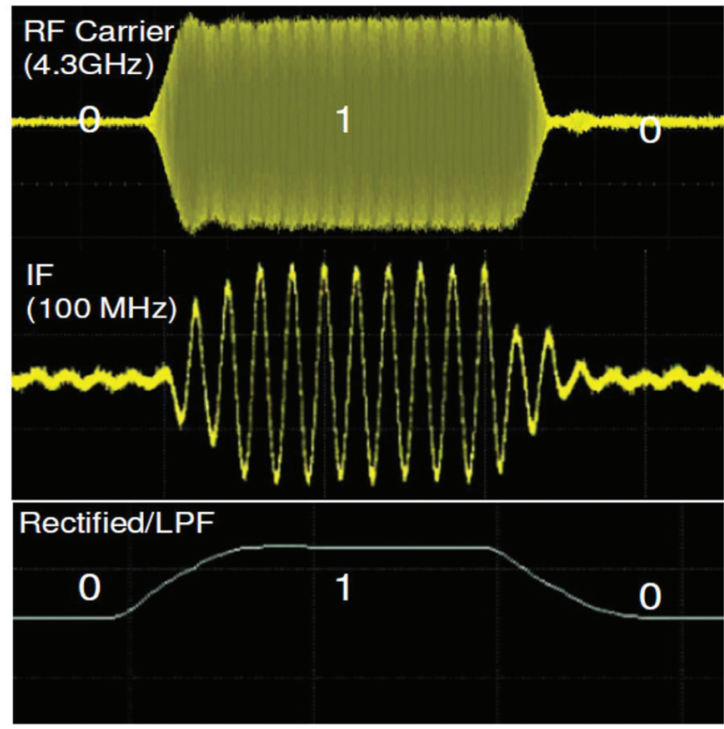

(b)

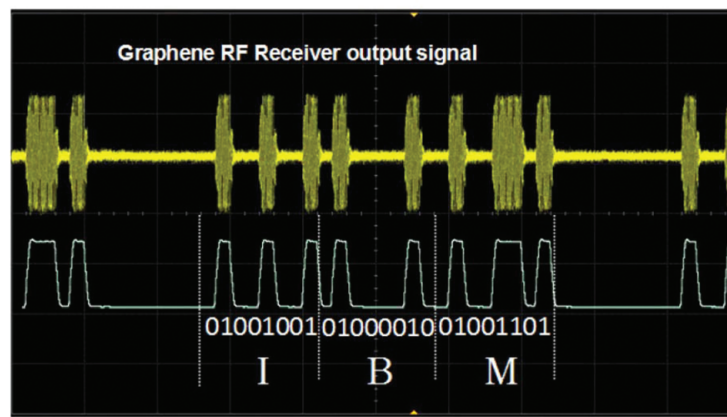

Fig. 16 (a) Measured waveforms of RF input signal amplitude modulated at a rate of $20 \mathrm{Mb} \mathrm{s}^{-1}$ (top), IF output signal (middle), and the restored binary code after rectifying and low-pass filtering IF signal (bottom). (b) A screenshot of receiver output waveforms taken from the oscilloscope, with LO power of $-2 \mathrm{dBm}$ at $4.2 \mathrm{GHz}$. (Taken from ref. 44. Reproduced with permission from Nature Publishing Group.)

Dirac voltage from the applied drain bias. Previously introduced amplifiers also exploited this characteristic., ${ }^{41,43}$

Fig. 17a shows the schematic diagram for the complementary-like graphene inverters. Fig. 17b depicts splitting of the Dirac point or charge neutrality point, due to the effect of $V_{\mathrm{DD}}$. A more detailed explanation is shown in Fig. 17c and d. When positive $V_{\mathrm{DD}}$ biases are applied, resistance curves of both FETs shift positively along the $V_{\mathrm{IN}}$ axis, in response to the additional superposition potential. Notably, the shift of FET2 is always larger than that of FET3, since FET2 is located upstream in the inverter loop and is affected by higher potential from $V_{\mathrm{DD}}$. By utilizing such an asymmetric shift, the CNP splitting can be controlled electrically (Fig. 17c). In Fig. 17d, when $V_{\mathrm{DS}}$ increases, the potential of the channel increases and the effective potential difference between TG and the channel decreases. As a result, the channel deviates from the neutrality condition, and additional positive charges are induced. To introduce charges with opposite polarity and reneutralize the channel, the $V_{\text {TG }}$ should increase by roughly half the value of $V_{\mathrm{DS}}$, resulting in a positive shift of $I_{\mathrm{DS}}$ minimum along the $V_{\mathrm{TG}}$
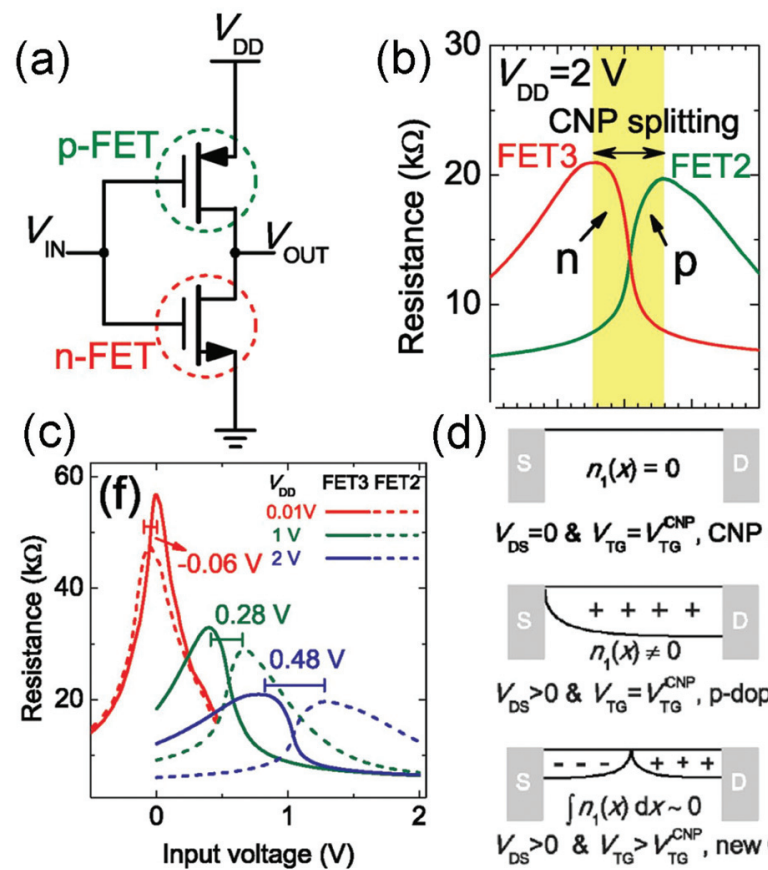

$V_{\mathrm{DS}}=0 \& V_{\mathrm{TG}}=V_{\mathrm{TQ}}^{\mathrm{CNP}}$, CNP state

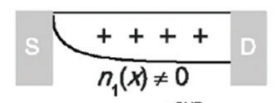

$V_{\mathrm{DS}}>0 \& V_{\mathrm{TG}}=V_{\mathrm{TG}}^{\mathrm{CNP}}, \mathrm{p}$-doping

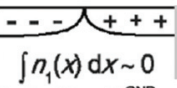$$
V_{\mathrm{DS}}>0 \& V_{\mathrm{TG}}>V_{\mathrm{TG}}^{\mathrm{CNP}} \text {, new CNP }
$$

Fig. 17 (a) Schematic diagram for the complementary-like graphene inverter. (b) The operating principle for the inverter consisted of FET2 and FET3. Once a CNP splitting between the FET pair is created by high $V_{\mathrm{DD}}$ along the input voltage axis, voltage inversion can be achieved in the region of CNP splitting. (c) The resistance behaviour of two FETs (in the inverter) under different $V_{\mathrm{DD}}$. (d) The charge distributions of the FET channel under varied $V_{\mathrm{DS}}$ and $V_{\mathrm{TG}}$ conditions. (Taken from ref. 49. Reproduced with permission from the American Chemical Society.)

axis. The inverters achieved voltage gains up to 7, indicating the potential for direct cascading of multiple devices.

Afterwards, the cascading of these inverters with appreciable voltage gain was successfully demonstrated by another group. ${ }^{50}$ The cascaded inverters resulted in full-channel gating with a low-frequency voltage gain of $A_{\mathrm{v}} \sim 5$. Such gain enabled logic inverters with the same voltage swing of $0.56 \mathrm{~V}$ at their input and output (Fig. 18).

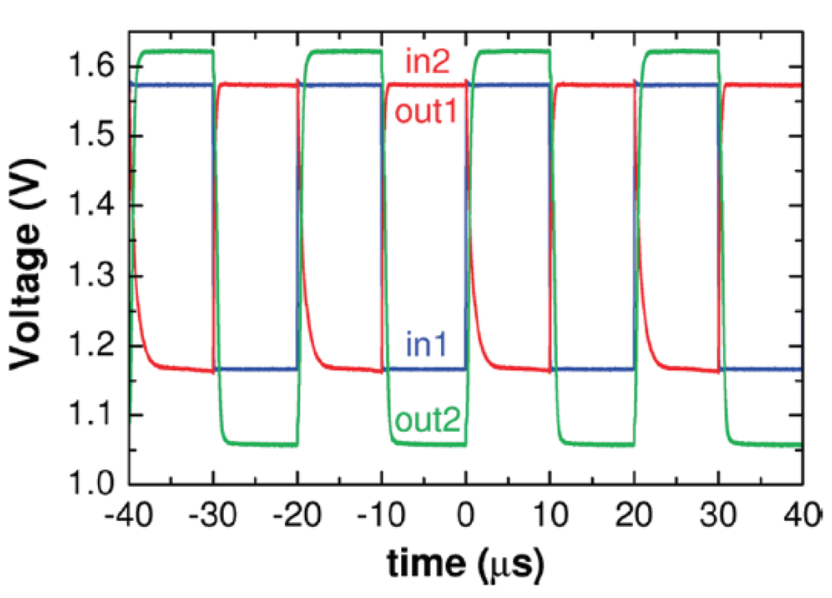

Fig. 18 Digital waveforms measured under ambient conditions in a cascade connection of two graphene inverters. (Taken from ref. 50. Reproduced with permission from the American Chemical Society.) 
Fig. 18 illustrates the digital waveforms measured under ambient conditions in a cascade connection of two graphene inverters. The supply voltage is $2.5 \mathrm{~V}$ and the frequency is $50 \mathrm{kHz}$.

\section{Passive components}

Another area of interest for graphene is its applications for passive components. Graphene is not only the strongest and the stiffest material in terms of tensile strength and elastic modulus, respectively; it is also extremely flexible, transparent, and conductive. The close-knit hexagonal crystal structure can also function as an effective barrier against most materials. These exceptional qualities are manifested due to graphene's covalent $\mathrm{sp}^{2}$ bonds. By combining its mechanical strength with unique electrical properties, graphene can be utilized to improve conventional electronic circuits ${ }^{47,53}$ or provide unique opportunities in niche areas, such as transparent, flexible electronics, ${ }^{37,52}$ displays, wearable electronics ${ }^{97}$ and biomedical skin-like devices. ${ }^{98}$

\section{Interconnects}

As dimension scaling continues in electronic circuits, many problems such as high resistance and reduced reliability plague copper-based interconnects. ${ }^{53,96}$ Although interconnects based on carbon nanotubes (CNTs) were also investigated for vertical vias, ${ }^{99}$ graphene has its advantages because it is compatible with conventional patterning and etching techniques.

Many researchers have investigated the application of graphene interconnects for several reasons. First, graphene is several times more conductive than copper of the same thickness. ${ }^{6}$ It is also known to sustain the highest amount of current density compared to any other known material. ${ }^{6}$ In addition, it is inherently immune to electromigration, ${ }^{47,96}$ and it is known to withstand very high temperatures. ${ }^{6}$ Lastly, graphene is known for its quenching effect of electrical resistance at high temperature. It was found that as the temperature increases from 300 to $500 \mathrm{~K}$, the resistance of single- and bilayer graphene interconnects drops by $30 \%$ and $70 \%$, respectively. ${ }^{51}$ The quenching and temperature dependence of the resistance were explained by the thermal generation of the electron-hole pairs and carrier scattering by acoustic phonons. $^{51}$

With these inherent advantages, high-speed, on-chip graphene interconnects that operate at frequencies up to $1.3 \mathrm{GHz}$ were integrated with $0.25 \mu \mathrm{m}$ technology CMOS ring oscillator. ${ }^{47}$ In the circuit diagram (Fig. 19a), the line is terminated by a termination resistor $R_{\mathrm{T}}=20 \mathrm{k} \Omega$ to a mid-supply voltage $V_{\mathrm{DD}} / 2$. Here the $R \sim 0.5 R_{\mathrm{T}}$ is a value determined by the tradeoff between the speed improvement provided by the low-swing signalling, and the sensitivity of the differential amplifier at the receiving end. Hence, the signal swing at the receiver end reduced to $V_{\mathrm{DD}} \times R / R_{\mathrm{T}}$, whereas the speed increased by a factor of $R / R_{\mathrm{T}}$. (a)

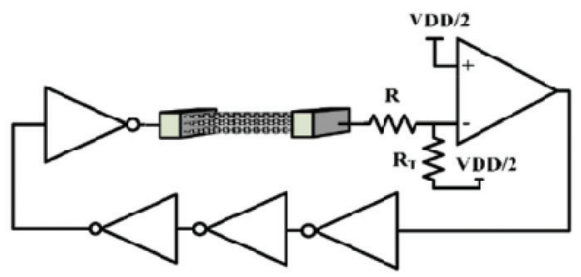

(b)

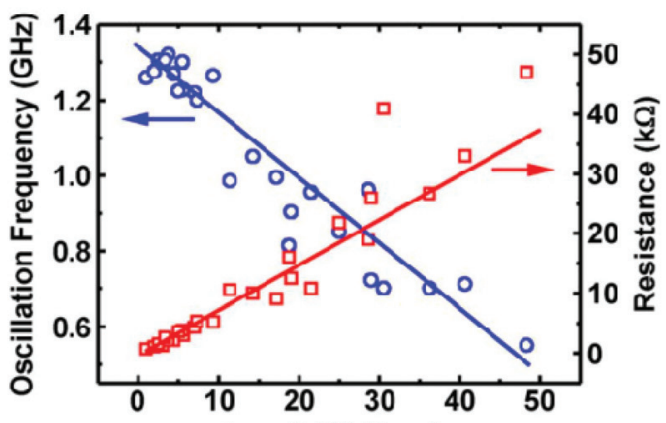

(c)

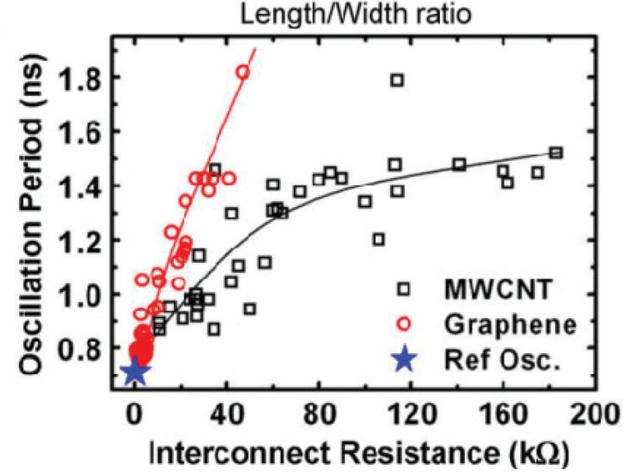

Fig. 19 (a) Circuit diagram of a five-stage ring oscillator with a differential amplifier at the receiver end of the inverter chain. (b) Oscillation frequency and resistance as functions of L/W ratio. Symbols represent experimental data and bold lines are visual guides. (c) Oscillation period as a function of interconnect resistance for the CMOS reference interconnects (aluminium), MWCNTs, and graphene. Symbols represent experimental data and bold lines are visual guides. (Taken from ref. 47. Reproduced by permission of the Institute of Electrical and Electronics Engineers (IEEE).)

The circuit oscillation frequency is directly correlated with the interconnect width and inversely correlated with the length/width ratio (Fig. 19b). Oscillation periods as a function of interconnect resistance for the reference interconnects (aluminium), MWCNTs, and graphene are shown in Fig. 19c. Graphene offers a higher oscillation frequency because of its lower resistivity. However, at the same resistance value, the MWCNT has superior signal delay performance. This is due to the larger total capacitance associated with wider graphene strips.

Although the demonstration of graphene interconnects at such high frequency is encouraging, practical problems still remain due to the high growth temperature $\left(\sim 1000{ }^{\circ} \mathrm{C}\right)$ of graphene. ${ }^{96}$ The high temperature is a concern since the graphene transfer process is not suitable for massive-scale fabrication of more than ten-level interconnects. A lower temperature process $\left(\sim 400^{\circ} \mathrm{C}\right)$ with direct deposition capability will be crucial to realize graphene-based interconnects. ${ }^{96}$ 


\section{Transparent conductors}

Graphene also shows interesting optical properties. Graphene absorbs a significant amount of light per layer (2.3\% per layer from ultraviolet to near infrared ${ }^{11}$ ). However, because it is only one atomic layer thick, graphene is highly transparent with transmittance of $97.7 \%$. In addition, graphene is also highly conductive due to its semi-metallic properties. ${ }^{4,53}$ The sheet resistance of a pristine layer is about $2 \mathrm{k} \Omega$ to $6 \mathrm{k} \Omega^{52,100}$ and can be as low as $125 \Omega$ with chemical doping. ${ }^{101}$ The sheet resistance value can be lowered even more by either stacking several graphene layers ${ }^{52}$ or fabricating a hybrid nanowire/ nanomesh structure ${ }^{102}$ with graphene. Several research groups have demonstrated graphene layers as a transparent conductive material that can compete with some of the oxide-based transparent conductive materials (e.g. indium tin oxide or ITO) that dominate the current market. ${ }^{103}$ ITO, however, suffers from high cost, material deterioration from ion diffusion, and brittleness, making it incompatible with flexible substrates. Graphene, on the other hand, shows great promise as a transparent conductor due to its high chemical resistivity, low manufacturing cost, and atomically thin, flexible structure. ${ }^{103,104}$

Fig. 20 shows the sheet resistance of bilayer graphene (BLG) stacks compared with indium oxide under a mechanical deformation. The BLG films were transferred onto polyethylene terephthalate (PET) flexible substrates and patterned for fourprobe measurement (Fig. 20a). Two samples of both BLG 1-transfer and BLG 4-transfer were tested in comparison with a

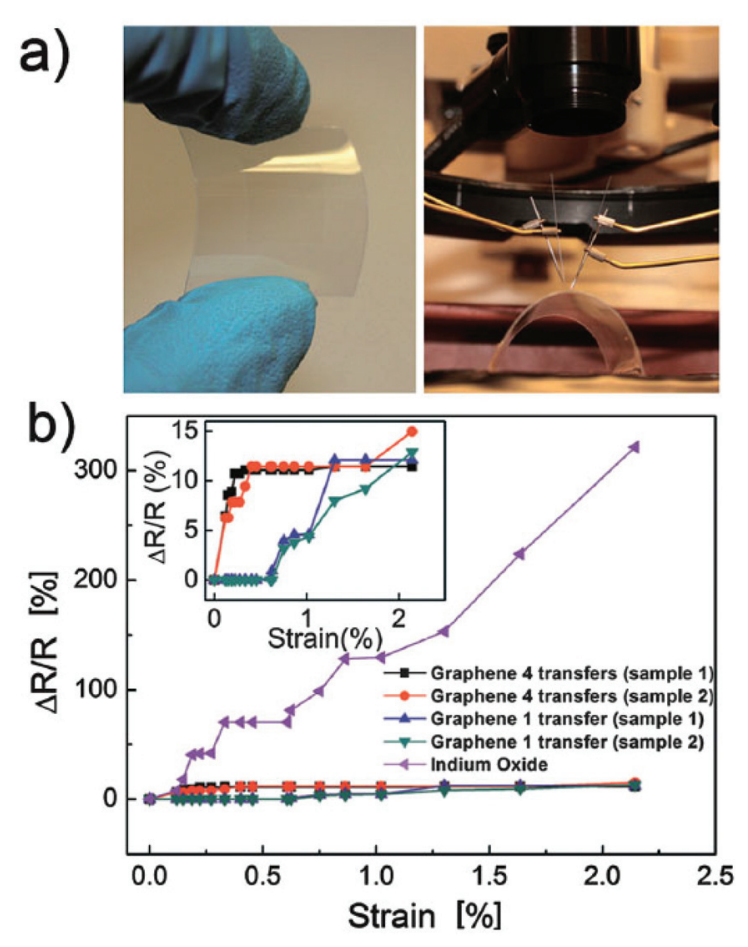

Fig. 20 (a) Photographs of a graphene film on the flexed PET substrate (left) and the measurement setup of strained substrates (right). (b) Variation in resistance of stacked BLG films and indium oxide films on $200 \mathrm{~mm}$ thick PET substrates as a function of strain values. (Taken from ref. 52. Reproduced by permission of The Royal Society of Chemistry.) commercial indium oxide on a PET substrate under bending conditions. Fig. 20b shows the relative change in sheet resistance versus strain due to bending. At $2.14 \%$ strain, the sheet resistance of the indium oxide sample increased by $321 \%$ while in the graphene samples it increased only by $10-15 \%$. The indium oxide sample shows a drastic change in sheet resistance due to its brittle nature while graphene samples are much more robust against mechanical stress.

\section{Inductors}

Since graphene-based field-effect transistors have already shown potential for analog/RF devices and interconnects, graphene can also be used to design low-loss on-chip inductors that are critical for RF circuits. For designing carbon-based inductors, graphene has a unique advantage over CNTs since it is more controllable from the fabrication point of view. ${ }^{47,53,96}$ This is due to the planar nature of graphene, which can be patterned using high-resolution lithography (Fig. 21a). Moreover, CNT-based inductors require metal contacts at each corner to connect CNT bundles (Fig. 21b), whereas no such contacts are needed for graphene-based inductors. ${ }^{53}$

The high frequency effects of graphene inductors have been thoroughly investigated by taking into account the kinetic inductance of graphene, the anomalous skin effect, and edge specularities (Fig. 21). ${ }^{53}$ Comparative studies with CNT- and copper-based inductors have also been done. The quality factors (Q-factor) of each design were compared using a modified $\pi$ model (Fig. 21c) of the on-chip inductors (Fig. 21d). A higher Q-factor is desirable because it leads to lower loss and higher frequency stability. ${ }^{94}$ With a fixed design on a low-loss (high-resistivity; $10 \Omega \mathrm{cm}$ ) substrate, the Q-factor of the graphene inductor shows an improvement of about $20 \%$ compared to that of copper. On the other hand, $50 \%$ improvement and $15 \%$ improvement were observed with respect to singlewalled CNT (with 1/3 metallic fraction) and multi-walled CNTbased inductors, respectively (Fig. 21d). An example of a graphene inductor patterned on graphene oxide films by using the direct femtosecond laser reduction process is shown in Fig. 21e. ${ }^{54}$

\section{Transition metal dichalcogenides- based circuits}

The study of transition metal dichalcogenides is currently a highly active area of research. Fundamental studies of transistor operations have been conducted on some of the more accessible TMDs such as $\mathrm{MoS}_{2}, \mathrm{WS}_{2}$, and $\mathrm{WSe}_{2}$ using mechanically exfoliated samples. Transistors with subthreshold slopes

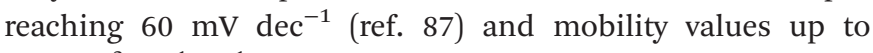
$250 \mathrm{~cm}^{2} \mathrm{~V}^{-1} \mathrm{~s}^{-1}$ (ref. 87) have already been demonstrated. However, to drive the field forward, the development of largescale synthesis and integration methods is crucial. Unlike graphene, which is usually synthesized on transition metals such as copper or nickel using $\mathrm{CVD},{ }^{18}$ several TMDs have been suc- 
(a)
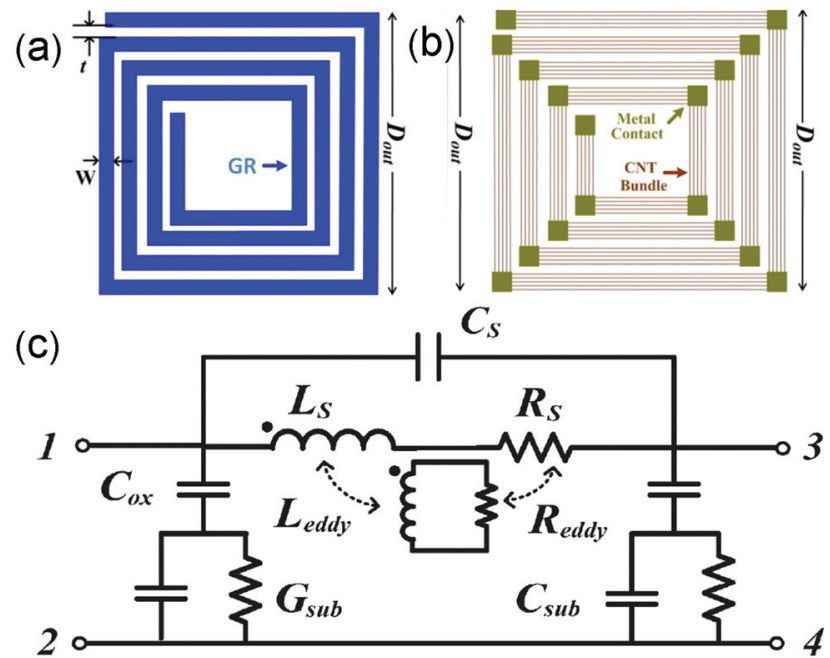

(d)

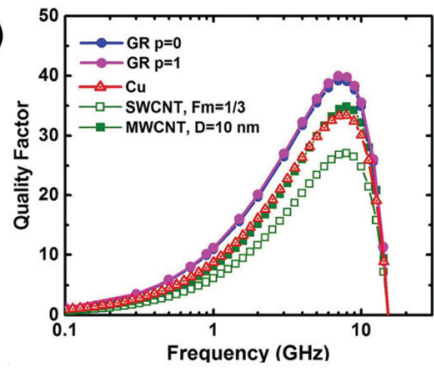

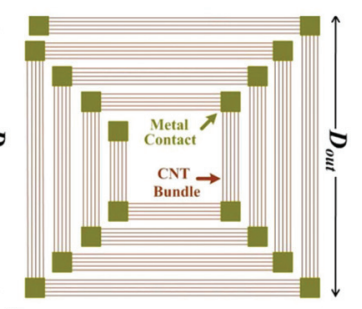

$C_{S}$

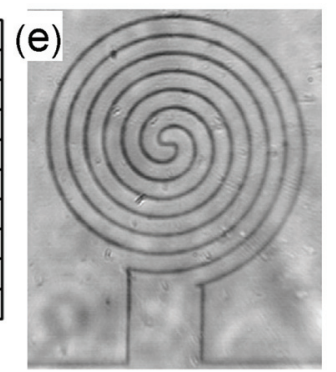

Fig. 21 (a) Schematic view of a four-turn spiral graphene-based inductor (b) CNT-based inductor. At each corner, there is a metal contact to connect CNT bundles. However, no such contacts are required for graphene-based inductors. (c) Modified $\pi$ model for on-chip spiral inductors. In this model, $L_{\mathrm{S}}$ and $R_{\mathrm{S}}$ are the series frequency-dependent inductance and resistance, respectively. $L_{\text {eddy }}$ and $R_{\text {eddy }}$ are the eddycurrent-induced parameters, and $C_{\mathrm{S}}, C_{\mathrm{ox}}$, and $C_{\text {sub }}$ are the inter-turn conductor capacitance, the oxide capacitance, and the substrate capacitance, respectively. (d) Q-factors of inductors based on graphene, $\mathrm{Cu}$, single-walled CNT, and multi-walled CNT interconnects as a function of frequency for low-loss $\left(\rho_{\text {sub }}=10 \Omega \mathrm{cm}\right.$ ) substrate. (Taken from ref. 53 . Reproduced by permission of the Institute of Electrical and Electronics Engineers (IEEE).) (e) Optical microscopy image of an inductor fabricated via direct imprinting on graphene oxide film using femtosecond laser reduction. (Taken from ref. 54. Reproduced by permission of Elsevier.)

cessfully synthesized on insulating layers such as glass, ${ }^{25}$ sapphire, ${ }^{25,27}$ and silicon dioxide. ${ }^{22,25-27,105}$ The capability to directly synthesize TMDs on top of a non-conducting substrate is an important step toward system-level integration of these 2D materials. However, it is important to note that the deposition temperature is still quite high $\left(650{ }^{\circ} \mathrm{C}-800{ }^{\circ} \mathrm{C}\right)$ and the films are polycrystalline. ${ }^{22,23,25-27,105}$ An effective way to synthesize higher quality, large-area single-crystalline films is still in high demand.

Integrated circuits such as inverters, ${ }^{55-58}$ small signal amplifiers, ${ }^{55}$ logic gates, ${ }^{56-58}$ a SRAM cell, ${ }^{57}$ and a 5-stage ring oscillator $^{57}$ were demonstrated using both exfoliated ${ }^{55-57}$ and CVD synthesized ${ }^{58} \mathrm{MoS}_{2}$ samples.

A small signal amplifier based on a single grain of exfoliated $\mathrm{MoS}_{2}$ samples is shown (Fig. 22). Unlike graphenebased transistors, $\mathrm{MoS}_{2}$ transistors can reach an on/off ratio of

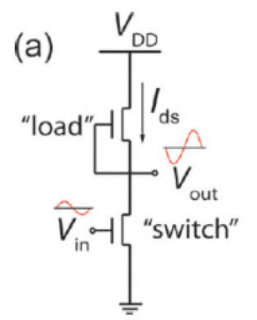

(b)

(c)

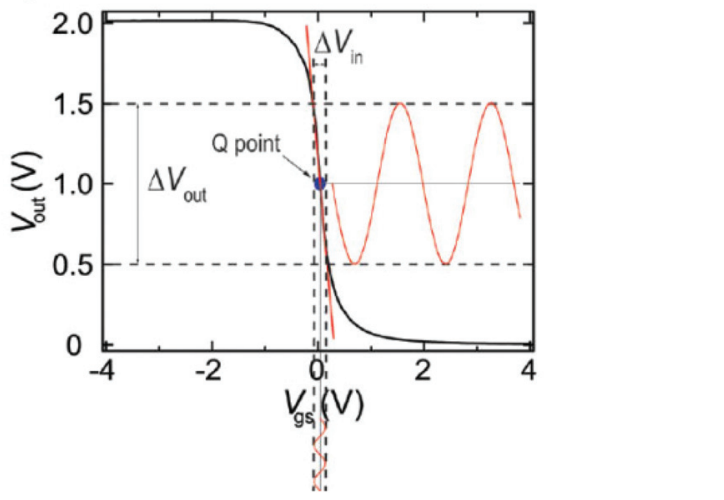

Fig. 22 (a) Schematic of an $\mathrm{MoS}_{2}$ amplifier in a common-source configuration. (b) Vertical cross-section of the amplifier. (c) Transfer characteristic of the amplifier. The "switch" transistor is biased at a quiescent point ( $Q$ point). The "load" transistor operates as resister at this point. A small AC signal of amplitude $\Delta V_{\text {in }} / 2$ is then superimposed on the gate bias and amplified. (Taken from ref. 55. Reproduced by permission of the American Institute of Physics.)

$10^{8}{ }^{2}$ The transconductance $g_{\mathrm{m}}$ reached a value of $12 \mu \mathrm{S}$ $\left(2.6 \mu \mathrm{S} \mu \mathrm{m}^{-1}\right)$ for $V_{\mathrm{ds}}=500 \mathrm{mV}$ and gate oxide of $\mathrm{HfO}_{2}(30 \mathrm{~nm})$ using optical lithography. ${ }^{55}$ The small signal amplifiers reached a gain of $\sim 4$ at $2 V_{\mathrm{DD}}$. The device configuration and the transfer characteristics are shown in Fig. 22.

An integrated circuit with inverters, logic gates, a SRAM cell, and a 5-stage ring oscillator was also demonstrated with exfoliated bilayer $\operatorname{MoS}_{2} \cdot{ }^{57}$ In Fig. 23a, a flip-flop SRAM circuit with $\mathrm{MoS}_{2}$ transistors can be set to logic state 1 (or 0 ) by applying a low (or high) voltage to the input. The output logic state stays at 0 or 1 after the switch to the input has been opened, thus serving as a non-volatile memory. In the NAND gate circuit using $\mathrm{MoS}_{2}$ transistors (Fig. 23b), the output of the circuit is close to logic state 1 when either or both of the inputs are at logic state 0 . The output is at logic state 0 only when both inputs are at logic state 1 (i.e. when both $\mathrm{MoS}_{2}$ FETs are conducting). The ring oscillator (Fig. 23c) was realized by cascading five inverter stages in a close loop. The transistor gate length $L_{\mathrm{g}}$ was $1 \mu \mathrm{m}\left(W / L_{\mathrm{g}} \sim 20\right)$. An extra inverter stage was used to synthesize the output signal by isolating the oscillator operation from the measurement setup. At $V_{\mathrm{dd}}=2 \mathrm{~V}$, the fundamental oscillation frequency is at $1.6 \mathrm{MHz}$, corresponding to a propagation delay of $\tau_{\mathrm{pd}}=1 /(2 n f)=62.5 \mathrm{~ns}$ per stage, where $n$ is the number of stages and $f$ is the fundamental oscillation frequency. Fig. 23d depicts the power spectrum of the output signal as a function of $V_{\mathrm{dd}}$. From left to right, $V_{\mathrm{dd}}$ is changed from $1.1 \mathrm{~V}$ to $2.0 \mathrm{~V}$ in steps of $0.1 \mathrm{~V}$. 

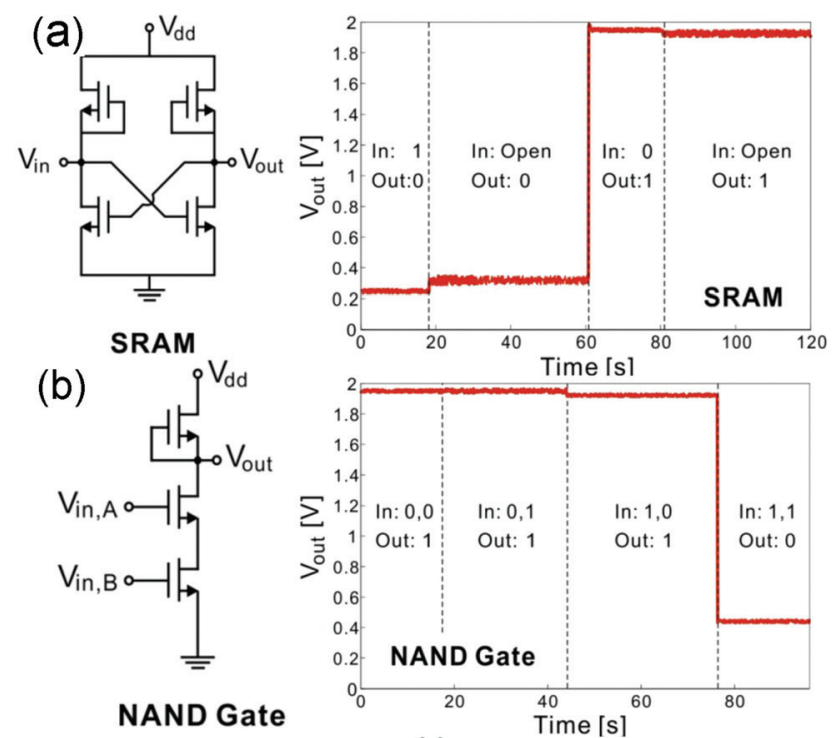

(C)
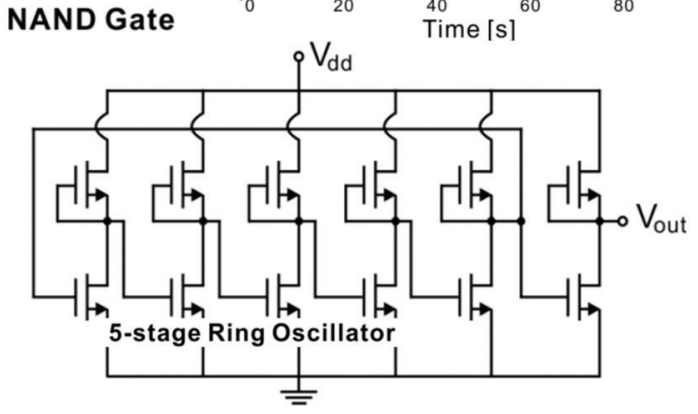

(d)

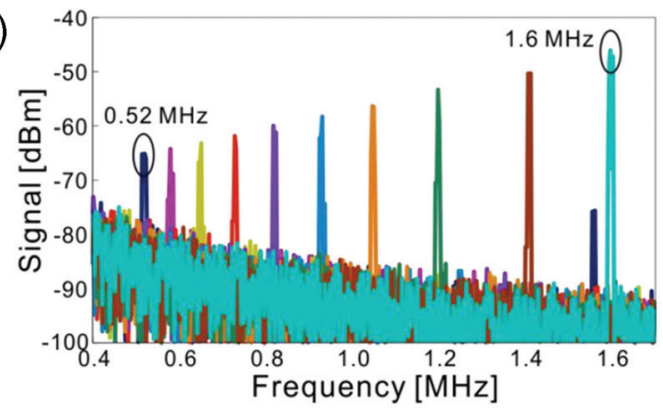

Fig. 23 (a) The schematics of the electronic circuits for SRAM, based on exfoliated bilayer $\mathrm{MoS}_{2}$ transistors and its output voltage characteristics. A logic state 1 (or 0 ) at the input voltage can set the output voltage to logic state 0 (or 1). (b) The schematics of the electronic circuits for NAND gate based on exfoliated bilayer $\mathrm{MoS}_{2}$ transistors, and its output voltage. A low voltage below $0.5 \mathrm{~V}$ represents a logic state 0 and a voltage close to $2 \mathrm{~V}$ represents a logic state 1 . (c) Schematic of the electronic circuit of the 5-stage ring oscillator. The first five inverter stages form the positive feedback loop, and the last inverter serves as the synthesis stage. (d) The power spectrum of the output signal as a function of $V_{\text {dd }}$. From left to right, $V_{\text {dd }}$ is changed from $1.15 \mathrm{~V}$ and 1.2 to $2.0 \mathrm{~V}$ in step of $0.1 \mathrm{~V}$. (Taken from ref. 57. Reproduced by permission of the American Chemical Society.)

\section{Conclusions and perspectives}

An important advantage of a two-dimensional structure compared to other nanomaterials is the straightforward integration with existing top-down fabrication processes. Although other zero-dimensional and one-dimensional nanostructures were discovered early and investigated for a longer period of time, the difficulty in controlling the exact position and the concentration of these individual nanomaterials was a major obstacle in commercialization for integrated electronics. ${ }^{8}$ For example, the chirality (directionality of graphitic hexagonal lattice) and the thickness of one-dimensional carbon nanotubes were very important as they determine the bandgap and the electronic behaviour of individual nanotubes. ${ }^{72}$ Controlling these parameters in carbon nanotubes still remains a key challenge, even after two decades of intense study since their discovery. ${ }^{106,107}$ On the other hand, with 2D materials, a facile integration of bottom-up nanomaterial synthesis with top-down lithographic fabrication is possible. After $2 \mathrm{D}$ materials are transferred onto a substrate, a conventional semiconductor fabrication process can be used to manufacture various devices. This property also allows seamless integration of the current state-of-the-art fabrication technology with 2D materials.

Based on the 2013 International Technology Roadmap for Semiconductors (ITRS) executive summary, the advancement of electronic technology can be categorized into two directions: More Moore (elements that scale according to the traditional "Moore's Law") and More than Moore (elements that add new functionalities [non-CMOS] that do not typically scale or behave according to the "Moore's Law"). Fig. 24 illustrates the potential applications and the challenges associated with the exploitation of the $2 \mathrm{D}$ materials in these two distinctive directions.

First, these materials can be utilized as a key component for the continuation of the Moore's law (More Moore, Fig. 24). From the scaling theory, a thin channel is required to minimize the short-channel effects. ${ }^{8}$ When other semiconducting materials are thinned down to such extreme $(<4 \mathrm{~nm})$ thickness, severe performance degradation arises from the surface states and the atomic thickness fluctuation. ${ }^{59-61}$ In contrast, naturally thin, inert $2 \mathrm{D}$ films with no dangling bonds can be highly effective in the lateral scaling (e.g. transistor scaling) and the vertical scaling (e.g. stacking of electronic components) of the electronic components. ${ }^{8,47,108}$ Ultrathin but highly functional interconnects and dielectrics are also essential to the continuation of Moore's law.

Second, with so many unique features available from different sets of 2D materials with various thicknesses, myriad possibilities emerge for unique functionalities (More than Moore, Fig. 24). This is especially true for components that require atomically sharp interfaces and smoothness, such as superlattices/heterostructure FETs, ${ }^{95}$ optoelectronic components, ${ }^{1,95,103}$ and photovoltaics. ${ }^{109}$ Various atomic layered heterostructures with 2D building blocks exhibited highly interesting characteristics due to quantum confinement and low density of states. For example, several heterostructures were used to make extremely thin $(<10 \mathrm{~nm})$ vertical transistors that depend on the modulation of barrier heights through a vertical E-field. ${ }^{110,111}$ In addition, Van Hove singularities in the electronic density of states of TMDs result in enhanced lightmatter interactions, leading to enhanced photon absorption and electron-hole creation. ${ }^{109}$ Consequently, extremely 

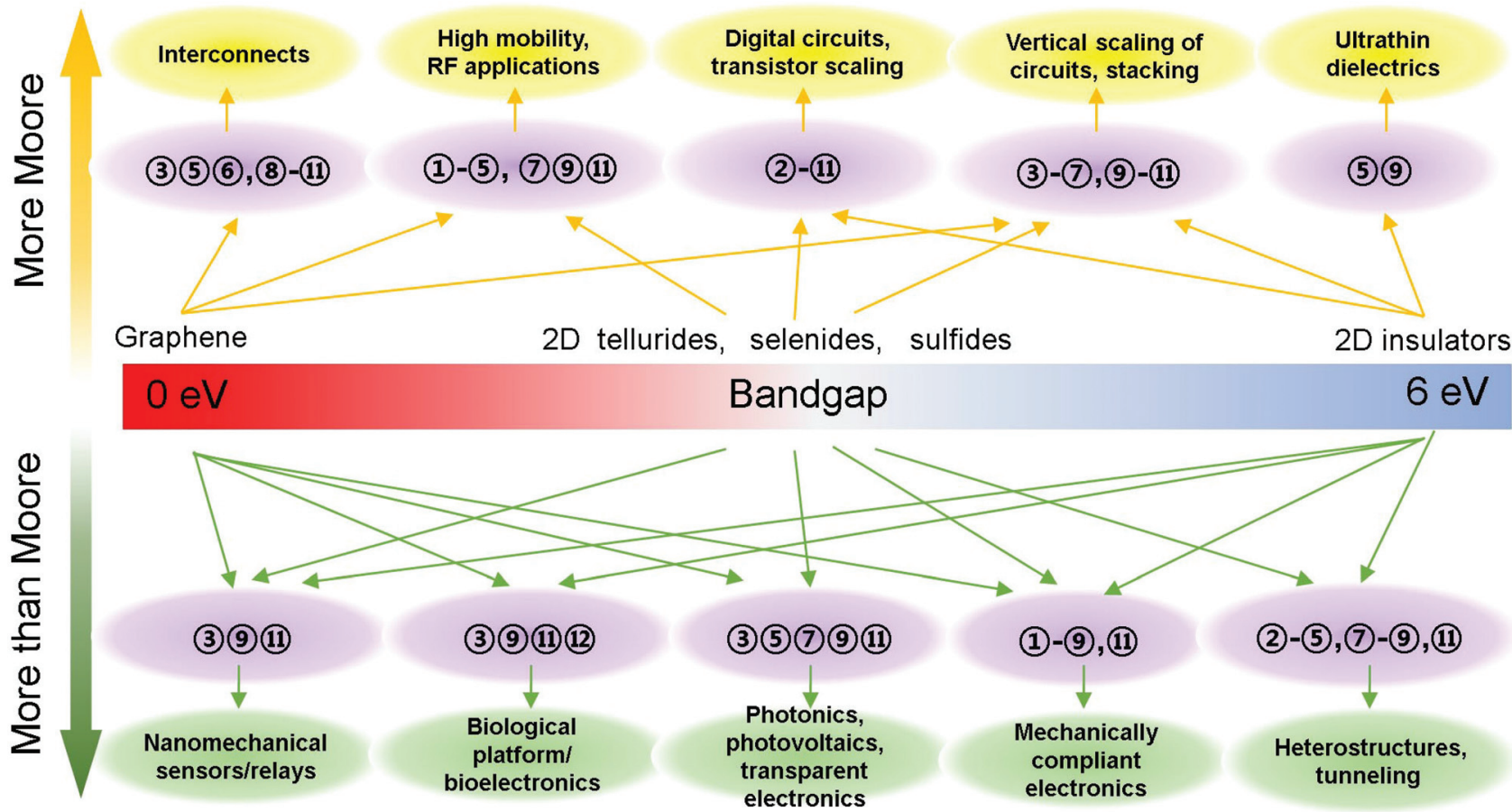

Fig. 24 Potential applications for 2D materials and the associated challenges. (1) The lack of current saturation and low gain of graphene transistors. (2) Relatively low mobility $\left(<200 \mathrm{~cm}^{2}\right)$ of $2 \mathrm{D}$ transition metal dichalcogenides. (3) Cleaning method to remove polymer/PR residue without using $\mathrm{O}_{2}$ plasma. (4) Pinhole-free dielectric formation. (5) Single crystalline/larger grain size (6) reusable substrate, waste handling. (7) Control of contact resistance/Schottky barrier/Fermi level pinning. (8) Effective doping methods stable in air and/or hermetic packaging. (9) Reducing defects from thermal coefficient mismatch of the film and the substrate during CVD. (10) Low temperature $\left(<400{ }^{\circ} \mathrm{C}\right)$ CVD synthesis for direct growth and integration. (11) Clean, repeatable interface after wet/dry transfer or direct synthesis without using metal catalyst. (12) Toxicity.

efficient photovoltaic (external quantum efficiency $>30 \%)^{109}$ and ultrahigh gain $\left(>10^{8}\right)$ photodetectors ${ }^{112}$ based on $2 \mathrm{D}$ heterostructures were demonstrated.

Since these materials can also be transferred to an arbitrary substrate, the possibility of utilizing exotic substrates such as plastics opens up doors for novel applications in both electronics and photonics. ${ }^{1,103}$ The mechanical robustness, flexibility, and transparency of the material will be indispensable for niche applications such as ultrathin displays, ${ }^{103}$ mechanically compliant electronics, ${ }^{37,52}$ photovoltaics, ${ }^{1,103}$ biomedical applications, ${ }^{113}$ transparent electronics, ${ }^{34}$ and nano-mechanical systems. ${ }^{114}$

Finally, several key challenges need to be addressed before 2D materials can be fully adopted into the mainstream technology. First, the growth temperature of these materials usually exceeds $600{ }^{\circ} \mathrm{C}^{22,25,27}$ Such a high temperature process is not always compatible with various fabrication steps. Furthermore, although film transfer is possible, a meticulous cleaning process is necessary during the wet transfers to achieve high quality interfaces. ${ }^{77}$ After the transfer and the following lithography steps, removing polymer residue without using $\mathrm{O}_{2}$ plasma is another challenge. $\left(\mathrm{O}_{2}\right.$ plasma is known to etch 2D materials. ${ }^{37,58}$ ) Second, either single-crystalline films or polycrystalline films with large grain boundaries are desirable for most electronics. Currently, most large-area films are polycrystalline with small grain boundaries, ${ }^{22,26,27,105}$ and most single-crystalline films are discontinuous (i.e. flakes). ${ }^{25,105}$ Defect control during the high temperature synthesis phase is also difficult. For example, the thermal expansion coefficient mismatch between the film and the substrate will result in residual thermal stress on the $2 \mathrm{D}$ films, which is responsible for defects and wrinkles. ${ }^{115}$ Third, forming good ohmic contacts with extremely thin semiconductors is a great challenge. ${ }^{116-119}$ Many TMDs (e.g. $\mathrm{MoS}_{2}$ ), being large bandgap materials, aggravate the problem. ${ }^{116}$ A promising method for assembling a superior ohmic contact with TMDs (e.g. $\left.\mathrm{MoS}_{2}\right)$ is to use graphene as the contact material. ${ }^{120,121}$ Not only was the barrier height of the graphene/ $\mathrm{MoS}_{2}$ interface determined to be lower than the popular Ti/MoS 2 contact, ${ }^{121}$ but the E-fieldinduced Fermi level control via the gate ${ }^{120}$ was found to be a promising way to produce a near-ohmic contact with higher on-state current. Fourth, doping these materials is not trivial since conventional interstitial doping will introduce defects. An alternative method is to exploit the charge transfer characteristics of different adsorbates. Various gas molecules were found to have a profound effect on the electrical transport properties of 2D films. ${ }^{92,122}$ For example, $\mathrm{H}_{2}, \mathrm{O}_{2}$, and $\mathrm{H}_{2} \mathrm{O}$ were found to work as charge acceptors in $\mathrm{MoS}_{2}$ after adsorption. Gases such as $\mathrm{NH}_{3}$ were found to be charge donors. ${ }^{122}$ However, retaining the gas molecules and their charge transfer qualities in ambient air over a prolonged time was found to be difficult to achieve. ${ }^{119}$ In addition, the various unintentional 
surface adsorbates from the environment may limit the performance of 2D films. Fifth, the highly inert surfaces of 2D materials render it difficult to form a pinhole-free dielectric with atomic layer deposition. ${ }^{123} \mathrm{~A}$ seed layer using $\mathrm{PVD}^{124}$ or spin coating ${ }^{123}$ is sometimes necessary, and optimization of this multilayer dielectric is required. Some of the mentioned challenges are much more critical in certain applications. An overview illustrated in Fig. 24 addresses some of the more pressing challenges for different applications.

It is difficult to determine specific timelines for each of these applications with many practical issues still waiting to be addressed. However, dealing with these issues is currently an active area of research, and recent progress shows great promise. ${ }^{87,117,118,125,126}$ It is safe to assume that initial applications will consist of both 2D materials and conventional bulk materials. However, an ultra-thin electronic system exclusively assembled with $2 \mathrm{D}$ materials ${ }^{127}$ is also plausible since various $2 \mathrm{D}$ materials with different electronic properties are now available. With the continued improvements in large-scale synthesis and integration, 2D materials will be a vital component for advanced circuits and systems in the near future.

\section{Acknowledgements}

Acknowledgement is made to the National Science Foundation Scalable Nanomanufacturing Program (DMR-1120187) and NSF CAREER Award (ECCS-1254468).

\section{Notes and references}

1 Q. H. Wang, K. Kalantar-Zadeh, A. Kis, J. N. Coleman and M. S. Strano, Nat. Nanotechnol., 2012, 7, 699-712.

2 B. Radisavljevic, A. Radenovic, J. Brivio, V. Giacometti and A. Kis, Nat. Nanotechnol., 2011, 6, 147-150.

3 R. Mas-Balleste, C. Gomez-Navarro, J. Gomez-Herrero and F. Zamora, Nanoscale, 2011, 3, 20-30.

4 A. K. Geim and K. S. Novoselov, Nat. Mater., 2007, 6, 183191.

5 K. Novoselov, A. K. Geim, S. Morozov, D. Jiang, M. Katsnelson, I. Grigorieva, S. Dubonos and A. Firsov, Nature, 2005, 438, 197-200.

6 A. K. Geim, Science, 2009, 324, 1530-1534.

7 M. Chhowalla, H. S. Shin, G. Eda, L.-J. Li, K. P. Loh and H. Zhang, Nat. Chem., 2013, 5, 263-275.

8 F. Schwierz, Nat. Nanotechnol., 2010, 5, 487-496.

9 K. S. Novoselov, A. K. Geim, S. Morozov, D. Jiang, Y. Zhang, S. Dubonos, I. Grigorieva and A. Firsov, Science, 2004, 306, 666-669.

10 X. Du, I. Skachko, A. Barker and E. Y. Andrei, Nat. Nanotechnol., 2008, 3, 491-495.

11 R. Nair, P. Blake, A. Grigorenko, K. Novoselov, T. Booth, T. Stauber, N. Peres and A. Geim, Science, 2008, 320, 1308-1308.
12 W. Wu, L. A. Jauregui, Z. Su, Z. Liu, J. Bao, Y. P. Chen and Q. Yu, Adv. Mater., 2011, 23, 4898-4903.

13 X. Ding, G. Ding, X. Xie, F. Huang and M. Jiang, Carbon, 2011, 49, 2522-2525.

14 C. Mattevi, H. Kim and M. Chhowalla, J. Mater. Chem., 2011, 21, 3324-3334.

15 C.-Y. Su, A.-Y. Lu, C.-Y. Wu, Y.-T. Li, K.-K. Liu, W. Zhang, S.-Y. Lin, Z.-Y. Juang, Y.-L. Zhong and F.-R. Chen, Nano Lett., 2011, 11, 3612-3616.

16 Q. Yu, L. A. Jauregui, W. Wu, R. Colby, J. Tian, Z. Su, H. Cao, Z. Liu, D. Pandey and D. Wei, Nat. Mater., 2011, 10, 443-449.

17 X. Li, W. Cai, J. An, S. Kim, J. Nah, D. Yang, R. Piner, A. Velamakanni, I. Jung, E. Tutuc, S. K. Banerjee, L. Colombo and R. S. Ruoff, Science, 2009, 324, 13121314.

18 S. Lee, K. Lee and Z. Zhong, Nano Lett., 2010, 10, 47024707.

19 A. Reina, X. Jia, J. Ho, D. Nezich, H. Son, V. Bulovic, M. S. Dresselhaus and J. Kong, Nano Lett., 2008, 9, 30-35.

20 W. Strupinski, K. Grodecki, A. Wysmolek, R. Stepniewski, T. Szkopek, P. Gaskell, A. Gruneis, D. Haberer, R. Bozek and J. Krupka, Nano Lett., 2011, 11, 1786-1791.

21 A. Guermoune, T. Chari, F. Popescu, S. S. Sabri, J. Guillemette, H. S. Skulason, T. Szkopek and M. Siaj, Carbon, 2011, 49, 4204-4210.

22 Y. H. Lee, X. Q. Zhang, W. Zhang, M. T. Chang, C. T. Lin, K. D. Chang, Y. C. Yu, J. T. W. Wang, C. S. Chang and L. J. Li, Adv. Mater., 2012, 24, 2320-2325.

23 W. Y. Lee, T. M. Besmann and M. W. Stott, J. Mater. Res., 1994, 9, 1474-1483.

24 J. Cheon, J. E. Gozum and G. S. Girolami, Chem. Mater., 1997, 9, 1847-1853.

25 S. Wu, C. Huang, G. Aivazian, J. S. Ross, D. H. Cobden and X. Xu, ACS Nano, 2013, 7, 2768-2772.

26 Y. Zhan, Z. Liu, S. Najmaei, P. M. Ajayan and J. Lou, Small, 2012, 8, 966-971.

27 K.-K. Liu, W. Zhang, Y.-H. Lee, Y.-C. Lin, M.-T. Chang, C.-Y. Su, C.-S. Chang, H. Li, Y. Shi and H. Zhang, Nano Lett., 2012, 12, 1538.

28 Y.-H. Lee, L. Yu, H. Wang, W. Fang, X. Ling, Y. Shi, C.-T. Lin, J.-K. Huang, M.-T. Chang and C.-S. Chang, Nano Lett., 2013, 13, 1852.

29 Y. Wu, Y.-m. Lin, A. A. Bol, K. A. Jenkins, F. Xia, D. B. Farmer, Y. Zhu and P. Avouris, Nature, 2011, 472, 74-78.

30 L. Liao, Y.-C. Lin, M. Bao, R. Cheng, J. Bai, Y. Liu, Y. Qu, K. L. Wang, Y. Huang and X. Duan, Nature, 2010, 467, 305-308.

31 F. Schwierz, Nature, 2011, 472, 41-42.

32 F. Schwierz, Proc. IEEE, 2013, 101, 1567-1584.

33 F. Schwierz, VLSI Technology, Systems and Applications (VLSI-TSA), 2011 International Symposium on, 2011, pp. 1-2.

34 H. Wang, D. Nezich, J. Kong and T. Palacios, IEEE Electron Device Lett., 2009, 30, 547-549. 
35 X. Yang, G. Liu, A. A. Balandin and K. Mohanram, ACS Nano, 2010, 4, 5532-5538.

36 Z. Wang, Z. Zhang, H. Xu, L. Ding, S. Wang and L.-M. Peng, Appl. Phys. Lett., 2010, 96, 173104.

37 S. Lee, K. Lee, C.-H. Liu, G. S. Kulkarni and Z. Zhong, Nat. Commun., 2012, 3, 1018.

38 H. Wang, A. Hsu, J. Wu, J. Kong and T. Palacios, IEEE Electron Device Lett., 2010, 31, 906-908.

39 Y.-M. Lin, A. Valdes-Garcia, S.-J. Han, D. B. Farmer, I. Meric, Y. Sun, Y. Wu, C. Dimitrakopoulos, A. Grill and P. Avouris, Science, 2011, 332, 1294-1297.

40 O. Habibpour, S. Cherednichenko, J. Vukusic, K. Yhland and J. Stake, IEEE Electron Device Lett., 2012, 33, 71-73.

41 E. Guerriero, L. Polloni, L. G. Rizzi, M. Bianchi, G. Mondello and R. Sordan, Small, 2012, 8, 357-361.

42 Y. Wu, K. A. Jenkins, A. Valdes-Garcia, D. B. Farmer, Y. Zhu, A. A. Bol, C. Dimitrakopoulos, W. Zhu, F. Xia and P. Avouris, Nano Lett., 2012, 12, 3062-3067.

43 S.-J. Han, K. A. Jenkins, A. Valdes Garcia, A. D. Franklin, A. A. Bol and W. Haensch, Nano Lett., 2011, 11, 36903693.

44 S.-J. Han, A. V. Garcia, S. Oida, K. A. Jenkins and W. Haensch, Nat. Commun., 2014, 5, 3086.

45 N. Harada, K. Yagi, S. Sato and N. Yokoyama, Appl. Phys. Lett., 2010, 96, 012102.

46 H.-Y. Chen and J. Appenzeller, Nano Lett., 2012, 12, 20672070.

47 X. Chen, D. Akinwande, K.-J. Lee, G. F. Close, S. Yasuda, B. C. Paul, S. Fujita, J. Kong and H.-S. Wong, IEEE Trans. Electron Devices, 2010, 57, 3137-3143.

48 S. L. Li, H. Miyazaki, M. V. Lee, C. Liu, A. Kanda and K. Tsukagoshi, Small, 2011, 7, 1552-1556.

49 S.-L. Li, H. Miyazaki, A. Kumatani, A. Kanda and K. Tsukagoshi, Nano Lett., 2010, 10, 2357-2362.

50 L. G. Rizzi, M. Bianchi, A. Behnam, E. Carrion, E. Guerriero, L. Polloni, E. Pop and R. Sordan, Nano Lett., 2012, 12, 3948-3953.

51 Q. Shao, G. Liu, D. Teweldebrhan and A. Balandin, Appl. Phys. Lett., 2008, 92, 202108.

52 S. Lee, K. Lee, C.-H. Liu and Z. Zhong, Nanoscale, 2012, 4, 639-644.

53 D. Sarkar, C. Xu, H. Li and K. Banerjee, IEEE Trans. Electron Devices, 2011, 58, 853-859.

54 Y. Zhang, L. Guo, S. Wei, Y. He, H. Xia, Q. Chen, H.-B. Sun and F.-S. Xiao, Nano Today, 2010, 5, 15-20.

55 B. Radisavljevic, M. B. Whitwick and A. Kis, Appl. Phys. Lett., 2012, 101, 043103.

56 B. Radisavljevic, M. B. Whitwick and A. Kis, ACS Nano, 2011, 5, 9934-9938.

57 H. Wang, L. Yu, Y.-H. Lee, Y. Shi, A. Hsu, M. L. Chin, L.-J. Li, M. Dubey, J. Kong and T. Palacios, Nano Lett., 2012, 12, 4674-4680.

58 H. Wang, L. Yu, Y.-H. Lee, W. Fang, A. Hsu, P. Herring, M. Chin, M. Dubey, L.-J. Li and J. Kong, APS March Meeting Abstracts, 2013, p. 7009.
59 K. Uchida, H. Watanabe, A. Kinoshita, J. Koga, T. Numata and S. Takagi, Electron Devices Meeting, 2002. IEDM'02. International IEEE, 2002, pp. 47-50.

60 L. Gomez, I. Aberg and J. Hoyt, IEEE Electron Device Lett., 2007, 28, 285-287.

61 K. Uchida and S.-i. Takagi, Appl. Phys. Lett., 2003, 82, 2916-2918.

62 V. Suntharalingam, R. Berger, J. A. Burns, C. K. Chen, C. L. Keast, J. M. Knecht, R. D. Lambert, K. L. Newcomb, D. M. O'Mara and D. D. Rathman, Solid-State Circuits Conference, 2005. Digest of Technical Papers. ISSCC. IEEE International, 2005, pp. 356-357.

63 R. S. Patti, Proc. IEEE, 2006, 94, 1214-1224.

64 J. Burns, L. McIlrath, C. Keast, C. Lewis, A. Loomis, K. Warner and P. Wyatt, Solid-State Circuits Conference, 2001. Digest of Technical Papers. ISSCC. IEEE International, 2001, pp. 268-269.

65 J. Kang, S. Tongay, J. Zhou, J. Li and J. Wu, Appl. Phys. Lett., 2013, 102, 012111.

66 A. Splendiani, L. Sun, Y. Zhang, T. Li, J. Kim, C.-Y. Chim, G. Galli and F. Wang, Nano Lett., 2010, 10, 1271-1275.

67 K. Watanabe, T. Taniguchi and H. Kanda, Nat. Mater., 2004, 3, 404.

68 R. R. Nair, W. Ren, R. Jalil, I. Riaz, V. G. Kravets, L. Britnell, P. Blake, F. Schedin, A. S. Mayorov and S. Yuan, Small, 2010, 6, 2877-2884.

69 M. I. Katsnelson, Mater. Today, 2007, 10, 20-27.

70 P. R. Wallace, Phys. Rev., 1947, 71, 622.

71 J. Slonczewski and P. Weiss, Phys. Rev., 1958, 109, 272.

72 A. C. Neto, F. Guinea, N. Peres, K. S. Novoselov and A. K. Geim, Rev. Mod. Phys., 2009, 81, 109.

73 G. W. Semenoff, Phys. Rev. Lett., 1984, 53, 2449-2452.

74 M. Freitag, Nat. Nanotechnol., 2008, 3, 455-457.

75 M. Trushin and J. Schliemann, Phys. Rev. Lett., 2007, 99, 216602.

76 M. Katsnelson, Eur. Phys. J. B, 2006, 51, 157-160.

77 S. Morozov, K. Novoselov, M. Katsnelson, F. Schedin, D. Elias, J. Jaszczak and A. Geim, Phys. Rev. Lett., 2008, 100, 016602.

78 K. Bolotin, K. Sikes, J. Hone, H. Stormer and P. Kim, Phys. Rev. Lett., 2008, 101, 096802.

79 E. V. Castro, H. Ochoa, M. Katsnelson, R. Gorbachev, D. Elias, K. Novoselov, A. Geim and F. Guinea, 2010, arXiv preprint arXiv:1008.2522.

80 A. S. Mayorov, R. V. Gorbachev, S. V. Morozov, L. Britnell, R. Jalil, L. A. Ponomarenko, P. Blake, K. S. Novoselov, K. Watanabe and T. Taniguchi, Nano Lett., 2011, 11, 23962399.

81 K. Nomura and A. MacDonald, Phys. Rev. Lett., 2007, 98, 076602.

82 M. Katsnelson and A. Geim, Philos. Trans. R. Soc. London, Ser. A, 2008, 366, 195.

83 R. Coehoorn, C. Haas, J. Dijkstra, C. Flipse, R. De Groot and A. Wold, Phys. Rev. B: Condens. Matter, 1987, 35, 6195 . 
84 H. Zhang, E. Bekyarova, J.-W. Huang, Z. Zhao, W. Bao, F. Wang, R. C. Haddon and C. N. Lau, Nano Lett., 2011, 11, 4047-4051.

85 D. B. Farmer, H.-Y. Chiu, Y.-M. Lin, K. A. Jenkins, F. Xia and P. Avouris, Nano Lett., 2009, 9, 4474-4478.

86 K. I. Bolotin, K. Sikes, Z. Jiang, M. Klima, G. Fudenberg, J. Hone, P. Kim and H. Stormer, Solid State Commun., 2008, 146, 351-355.

87 H. Fang, S. Chuang, T. C. Chang, K. Takei, T. Takahashi and A. Javey, Nano Lett., 2012, 12, 3788-3792.

88 A. L. Elías, N. Perea-López, A. s. Castro-Beltrán, A. Berkdemir, R. Lv, S. Feng, A. D. Long, T. Hayashi, Y. A. Kim and M. Endo, ACS Nano, 2013, 7, 5235-5242.

89 R. Coehoorn, C. Haas and R. De Groot, Phys. Rev. B: Condens. Matter, 1987, 35, 6203.

90 G. Eda, T. Fujita, H. Yamaguchi, D. Voiry, M. Chen and M. Chhowalla, ACS Nano, 2012, 6, 7311-7317.

91 C.-H. Lee, G.-H. Lee, A. M. van der Zande, W. Chen, Y. Li, M. Han, X. Cui, G. Arefe, C. Nuckolls and T. F. Heinz, 2014, arXiv preprint arXiv:1403.3062.

92 S. Tongay, J. Zhou, C. Ataca, J. Liu, J. S. Kang, T. S. Matthews, L. You, J. Li, J. C. Grossman and J. Wu, Nano Lett., 2013, 13, 2831.

93 M. Abbasi, R. Kozhuharov, C. Karnfelt, I. Angelov, I. Kallfass, P. Leuther and H. Zirath, IEEE Trans. Microw. Theory Tech., 2009, 57, 3134-3142.

94 T. H. Lee, The design of CMOS radio-frequency integrated circuits, Cambridge university press, 2004.

95 S. M. Sze and K. K. Ng, Physics of semiconductor devices, John Wiley \& Sons, 2006.

96 Y. Awano, Electron Devices Meeting (IEDM), 2009 IEEE International IEEE, 2009, pp. 1-4.

97 Y. Meng, Y. Zhao, C. Hu, H. Cheng, Y. Hu, Z. Zhang, G. Shi and L. Qu, Adv. Mater., 2013, 25, 2326-2331.

98 T. T. Tung, M. Castro, T. Y. Kim, K. S. Suh and J.-F. Feller, J. Mater. Chem., 2012, 22, 21754-21766.

99 M. Nihei, A. Kawabata, D. Kondo, M. Horibe, S. Sato and Y. Awano, Jpn. J. Appl. Phys., 2005, 44, 1626.

100 P. Blake, P. D. Brimicombe, R. R. Nair, T. J. Booth, D. Jiang, F. Schedin, L. A. Ponomarenko, S. V. Morozov, H. F. Gleeson and E. W. Hill, Nano Lett., 2008, 8, 1704-1708.

101 S. Bae, H. Kim, Y. Lee, X. Xu, J.-S. Park, Y. Zheng, J. Balakrishnan, T. Lei, H. R. Kim and Y. I. Song, Nat. Nanotechnol., 2010, 5, 574.

102 Y. Zhu, Z. Sun, Z. Yan, Z. Jin and J. M. Tour, ACS Nano, 2011, 5, 6472-6479.

103 F. Bonaccorso, Z. Sun, T. Hasan and A. Ferrari, Nat. Photonics, 2010, 4, 611-622.

104 J. K. Wassei and R. B. Kaner, Mater. Today, 2010, 13, 52-59.

105 S. Najmaei, Z. Liu, W. Zhou, X. Zou, G. Shi, S. Lei, B. I. Yakobson, J.-C. Idrobo, P. M. Ajayan and J. Lou, Nat. Mater., 2013, 12, 754-759.

106 H. Dai, Surf. Sci., 2002, 500, 218-241.
107 H. Wei, M. Shulaker, G. Hills, H.-Y. Chen, C.-S. Lee, L. Liyanage, J. Zhang, H.-S. P. Wong and S. Mitra Design, Automation \& Test in Europe Conference \& Exhibition (DATE), 2013 IEEE, 2013, p. 619.

108 W. Zhu, T. Low, Y.-H. Lee, H. Wang, D. B. Farmer, J. Kong, F. Xia and P. Avouris, Nat. Commun., 2014, 5, 3087.

109 L. Britnell, R. Ribeiro, A. Eckmann, R. Jalil, B. Belle, A. Mishchenko, Y.-J. Kim, R. Gorbachev, T. Georgiou and S. Morozov, Science, 2013, 340, 1311-1314.

110 L. Britnell, R. Gorbachev, R. Jalil, B. Belle, F. Schedin, A. Mishchenko, T. Georgiou, M. Katsnelson, L. Eaves and S. Morozov, Science, 2012, 335, 947-950.

111 H. Yang, J. Heo, S. Park, H. J. Song, D. H. Seo, K.-E. Byun, P. Kim, I. Yoo, H.-J. Chung and K. Kim, Science, 2012, 336, 1140-1143.

112 W. Zhang, C.-P. Chuu, J.-K. Huang, C.-H. Chen, M.-L. Tsai, Y.-H. Chang, C.-T. Liang, Y.-Z. Chen, Y.-L. Chueh and J.-H. He, Sci. Rep., 2014, 4, 3826.

113 Y. Zhang, T. R. Nayak, H. Hong and W. Cai, Nanoscale, 2012, 4, 3833-3842.

114 N. N. Klimov, S. Jung, S. Zhu, T. Li, C. A. Wright, S. D. Solares, D. B. Newell, N. B. Zhitenev and J. A. Stroscio, Science, 2012, 336, 1557-1561.

115 S. J. Chae, F. Güneş, K. K. Kim, E. S. Kim, G. H. Han, S. M. Kim, H. J. Shin, S. M. Yoon, J. Y. Choi and M. H. Park, Adv. Mater., 2009, 21, 2328-2333.

116 I. Popov, G. Seifert and D. Tománek, Phys. Rev. Lett., 2012, 108, 156802.

117 J.-R. Chen, P. M. Odenthal, A. G. Swartz, G. C. Floyd, H. Wen, K. Y. Luo and R. K. Kawakami, Nano Lett., 2013, 13, 3106-3110.

118 W. Liu, J. Kang, D. Sarkar, Y. Khatami, D. Jena and K. Banerjee, Nano Lett., 2013, 13, 1983-1990.

119 S. McDonnell, R. Addou, C. Buie, R. M. Wallace and C. L. Hinkle, ACS Nano, 2014, 8, 2880.

120 Y. T. Lee, K. Choi, H. S. Lee, S. W. Min, P. J. Jeon, D. K. Hwang, H. J. Choi and S. Im, Small, 2014, 12, 23562361.

121 J. Y. Kwak, J. Hwang, B. Calderon, H. Alsalman, N. Munoz, B. Schutter and M. G. Spencer, Nano Lett., 2014, 14, 4511-4516.

122 Q. Yue, Z. Shao, S. Chang and J. Li, Nanoscale Res. Lett., 2013, 8, 1.

123 F. Xia, D. B. Farmer, Y.-m. Lin and P. Avouris, Nano Lett., 2010, 10, 715-718.

124 A. Hsu, H. Wang, K. K. Kim, J. Kong and T. Palacios, IEEE Electron Device Lett., 2011, 32, 1008-1010.

125 S. Das, H.-Y. Chen, A. V. Penumatcha and J. Appenzeller, Nano Lett., 2012, 13, 100-105.

126 H. Fang, M. Tosun, G. Seol, T. C. Chang, K. Takei, J. Guo and A. Javey, Nano Lett., 2013, 13, 1991-1995.

127 T. Roy, M. Tosun, J. S. Kang, A. B. Sachid, S. Desai, M. Hettick, C. C. Hu and A. Javey, ACS Nano, 2014, 8, 6259. 